\title{
Evidence for an expanding corona based on spectral-timing modelling of multiple black hole $\mathrm{X}$-ray binaries
}

\author{
Zheng Cao ${ }^{\oplus, 1,2,3 \star}$ Matteo Lucchini ${ }^{\circledR},{ }^{1,4}$ Sera Markoff ${ }^{\oplus}, 1,5$ Riley M. T. Connors ${ }^{\oplus 6}$ \\ and Victoria Grinberg ${ }^{7}$ \\ ${ }^{1}$ Anton Pannekoek Institute for Astronomy, University of Amsterdam, Science Park 904, NL-1098 XH, Amsterdam, The Netherlands \\ ${ }^{2}$ SRON, Netherlands Institute for Space Research, Sorbonnelaan 2, NL-3584 CA, Utrecht, The Netherlands \\ ${ }^{3}$ Department of Astrophysics/IMAPP, Radboud University, P.O. Box 9010, NL-6500 GL, Nijmegen, The Netherlands \\ ${ }^{4}$ MIT Kavli Institute for Astrophysics and Space Research, MIT, 70 Vassar Street, Cambridge, MA NL-02139, USA \\ ${ }^{5}$ Gravitation Astroparticle Physics Amsterdam (GRAPPA) Institute, University of Amsterdam, Science Park NL-904, 1098 XH Amsterdam, The Netherlands \\ ${ }^{6}$ Cahill Center for Astronomy and Astrophysics, California Institute of Technology, Pasadena, CA NL-91125, USA \\ ${ }^{7}$ European Space Agency (ESA), European Space Research and Technology Centre (ESTEC), Keplerlaan 1, NL-2201 AZ Noordwijk, The Netherlands
}

Accepted 2021 October 19. Received 2021 September 28; in original form 2021 June 25

\begin{abstract}
Galactic black hole X-ray binaries (BHXBs) provide excellent laboratories to study accretion, as their relatively quick evolution allows us to monitor large changes in the in-flowing and/or out-flowing material over human time-scales. However, the details of how the inflow-outflow coupling evolves during a BHXB outburst remain an area of active debate. In this work, we attempt to probe the physical changes underlying the system evolution, by performing a systematic analysis of the multi-wavelength data of three BHXB sources: XTE J1752-223, MAXI J1659-152, and XTE J1650-500, during hard and hard-intermediate states. Using the power spectral hue which characterizes the X-ray variability properties, we identify several clusters of BHXB epochs and perform the joint multi-wavelength spectral modelling to test their commonality with a physical jet model. Under the assumption that the corona is related to the base of the jet, we find that the power spectral hue traces the variation of the coronal radius (from $\sim 10 R_{g}-\sim 40 R_{g}$ ) in multiple BHXBs at hard and hard-intermediate states, and that the data are consistent with moderately truncated accretion discs $\left(<25 R_{g}\right)$ during hard-intermediate states. We also find that all epochs of low disc reflection have high hue located near the hard-intermediate to soft-intermediate state transition, indicating that in these states the vertical extent of the corona and/or its bulk speed are increasing. Our results link the geometrical similarity in the corona among multiple BHXB sources to their timing characteristics, and probe the corona responding to the disc-jet interactions at hard and intermediate states during outbursts.
\end{abstract}

Key words: accretion, accretion discs - black hole physics - X-rays: binaries.

\section{INTRODUCTION}

Galactic low mass black hole X-ray binaries (BHXBs) are binary systems with a stellar-mass black hole accreting mass from its low mass companion star. The majority of Galactic BHXBs are transient X-ray sources that go from quiescence to outburst phases (e.g. Elvis et al. 1975; Tanaka \& Shibazaki 1996; Chen, Shrader \& Livio 1997; Homan \& Belloni 2005; Remillard \& McClintock 2006; Corral-Santana et al. 2016; Tetarenko et al. 2016). The outbursts can last weeks to months, usually exhibiting transitions between a well-defined series of X-ray spectral states (e.g. Nowak 1995; Esin, McClintock \& Narayan 1997; Méndez, Belloni \& van der Klis 1998; Homan \& Belloni 2005; Remillard \& McClintock 2006; Belloni 2010).

During a typical outburst, the X-ray spectrum of a source is initially at the hard state (HS), dominated by a hard power-law component as the luminosity rises from quiescence to highly luminous states. This power-law component is thought to be generated due to the inverse-

^E-mail: z.cao@sron.nl comptonization of soft-disc photons by hot electrons in a loosely defined 'compact corona'. Different proposed models for this corona include the innermost regions of a radiatively inefficient accretion flow (RIAF, e.g. Narayan \& Yi 1994; Esin et al. 1997; Yuan et al. 2007; Veledina, Poutanen \& Vurm 2013), an extended slab corona over the disc (e.g. Haardt \& Maraschi 1993; Haardt, Maraschi \& Ghisellini 1994), or a 'lamp-post', compact magnetized region above the disc and/or moving vertically above the black hole (e.g. Matt et al. 1992; Martocchia \& Matt 1996; Beloborodov 1999; Merloni \& Fabian 2002). A physical realization of this model would be if the lamp-post is associated with the base of the jet (e.g. Markoff \& Nowak 2004; Markoff, Nowak \& Wilms 2005; Maitra et al. 2009; Dauser et al. 2013; Fürst et al. 2015; Miller et al. 2015; Lucchini et al. 2021). As the accretion rate increases, the X-ray spectrum of the source softens, becoming increasingly dominated by the thermal emission of a geometrically thin, radiatively efficient accretion disc (Shakura \& Sunyaev 1973), and the hard power-law component softens and fades. This is the so-called 'soft state' (SS). Eventually, the source will again decrease in luminosity and transition back to the HS before returning to quiescence. The transitional spectral states are referred to as the hard/soft intermediate states (HIMS/SIMS) 
(Homan \& Belloni 2005; Belloni 2010). Roughly 40 percent of outbursts fail to reach the SS; these are termed 'failed' outbursts (Tetarenko et al. 2016).

Compact, magnetized jets are one of two types of characteristic outflows associated with BHXB outbursts. Jets are collimated relativistic streams of plasma launched from the inner accretion region. Particles are also accelerated within BHXB jets, similarly to AGN (active galactic nuclei, Markoff, Falcke \& Fender 2001a; Fender, Maccarone \& Van Kesteren 2005; Romero \& Orellana 2005; Bosch-Ramon, Romero \& Paredes 2006; Kylafis et al. 2012; Malzac 2013; Kantzas et al. 2021), leading to synchrotron emission by relativistic electrons. The stratification of optically-thick regions generates a flat/inverted radio-through-IR spectrum, which is one of the hallmarks of the presence of jets (Blandford \& Königl 1979; Hjellming \& Johnston 1988; Corbel et al. 2000; Fender et al. 2000; Fender, Belloni \& Gallo 2004). During a typical BHXB outburst, compact jets are present during the HS, and suppressed during the SS/quenched before the SS (Tananbaum et al. 1972; Fender et al. 1999, 2004; Gallo, Fender \& Pooley 2003; Belloni 2010, but see Drappeau et al. 2017).

Although the underlying physics is not yet understood, the coupling between inflowing and outflowing material results in a correlation between the radio and X-ray luminosity (e.g Hannikainen et al. 1998; Corbel et al. 2000, 2003, 2013; Gallo et al. 2003, 2014). This correlation can be extended to accreting supermassive black holes in jetted AGN by adding a term accounting for the black hole mass (Merloni, Heinz \& Di Matteo 2003; Falcke, Körding \& Markoff 2004; Plotkin et al. 2012); this extension is referred to as 'the fundamental Plane of black hole accretion'. This connection between radio and $\mathrm{X}$-ray emission, together with other observational similarities in such as the X-ray variability or the broadband spectral energy distributions (e.g. McHardy et al. 2006; Markoff et al. 2008, 2015), supports the idea that (at least to first order) the accretion physics can be scaled between black holes across the mass scale. However, the viscous time-scale in AGN is on the order of $\approx 10^{3-4} \mathrm{yr}$; therefore, we can only rely on BHXBs to probe the jet-disc coupling of the same source at vastly different accretion rates.

The X-ray light curves of BHXBs show variability across a wide range of time-scales. The information carried by this short-term variability can be used to complement spectral data to characterize the accretion flow, typically by employing Fourier techniques (e.g. Van der Klis 1989; Wijnands \& van der Klis 1999; Klein-Wolt \& Van Der Klis 2008; Uttley et al. 2014). The continuum shape of the power spectral density (hereafter PSD) is strongly correlated with the X-ray spectral states, possibly because both are driven by the geometry of the system (e.g. Done \& Kubota 2006; Done, Gierliński \& Kubota 2007; Belloni 2010; Ingram \& Done 2012; Rapisarda, Ingram \& van der Klis 2017; Ingram \& Motta 2019). Understanding both the spectral and timing properties of accreting black holes and how they are related to each other is very important since they are simultaneously produced by the same accretion process and together can provide additional independent constraints to models of black hole accretion.

Heil et al. (2015a) introduced the new concept of power spectral 'power-colours', defined as the ratio of the variances between two different Fourier frequency bands in the PSD, characterizing the shape of the PSD independently of its normalization (power colour index 1 $=$ variance in $0.25-2.0 \mathrm{~Hz} / 0.0039-0.031 \mathrm{~Hz}$, and power colour index 2 = variance in $0.031-0.25 \mathrm{~Hz} / 2.0-16.0 \mathrm{~Hz}$, hereafter PC1 and PC2). By performing the analysis of 12 different BHXB sources, Heil et al. (2015a) found that power colour provides a complementary phase-

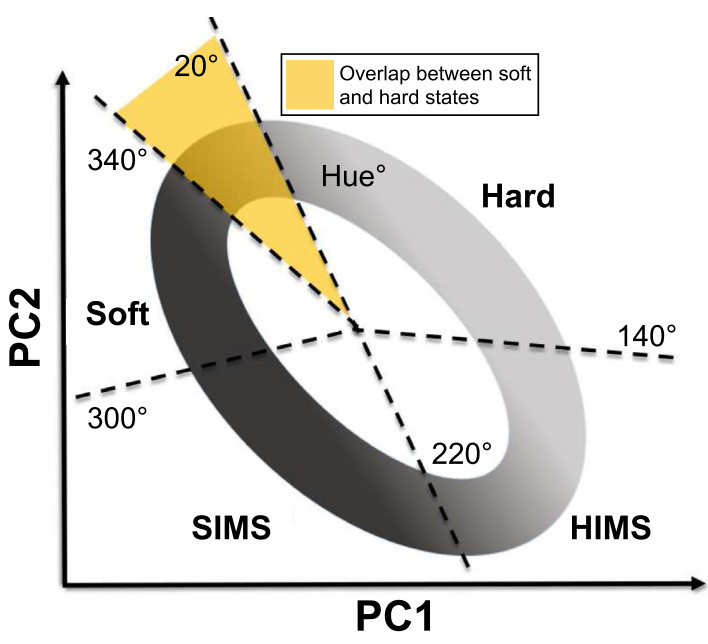

Figure 1. A conceptual illustration of the power-colour hue diagram, taken from Connors et al. (2019) based upon Heil, Uttley \& Klein-Wolt (2015a). Both axes are in $\log$ scale. Hue is defined as the angular position on the diagram where $0^{\circ}$ corresponds to the semi-major axis at $45^{\circ}$ to the $\mathrm{x}$ - and y-axes. Dotted lines mark the regions typically corresponding to spectral states: soft, hard, hard intermediate state(HIMS), and soft intermediate state (SIMS); soft and hard states overlap in the top left of the diagram due to similar shapes in power density spectra, which can be distinguished only by spectral analysis. A typical BHXB outburst will start in quiescence, a low luminosity extension of the hard state, and evolve clockwise to the soft state, and then move anti-clockwise back to the hard states (Heil et al. 2015a).

space to the spectrally-based hardness-intensity diagram (HID) in order to trace the evolution of BHXB outbursts via their angular positions on the power-colour diagram (PC1 versus PC2), defined as the 'hue' angles (illustrated in Fig. 1). The correlation between the spectral hardness and the hue is shared among different sources, but the physical reason behind this empirical correlation is largely unknown. In this way, a typical outburst will start from the HS with a small hue angle (at the top left of the PC1/PC2 diagram), evolve clockwise through the HIMS/SIMS to the SS with a large hue on the power-colour diagram (PCD), and then return to quiescence following a similar counter-clockwise track.

In this paper, we continue the exploration begun in Connors et al. (2019) and Lucchini et al. (2021), attempting to link the $\mathrm{X}$-ray timing and multi-wavelength properties within the context of outflow-dominated BHXB models, including a more detailed coronal region. We perform a systematic study of multiple BHXB sources, combining the X-ray variability classification scheme of Heil et al. (2015a) with broadband spectral energy distribution (SED) modelling during both hard states and hard-to-soft transitions. The goal of our modelling effort is to identify a consistent picture of the system evolution throughout outbursts. We do this by trying to identify possible trends in the geometry of the X-ray emitting region with respect to the spectral and timing evolution of the sources.

The paper is structured as follows. In Section 2, we describe the source selection and the data used in this work. In Section 3, we discuss our disc + jet model used in the spectral analysis, along with our assumptions. In Section 4, we present both the results of our broadband spectral fits and X-ray timing analysis, as well as the trends in the geometrical parameters we find in the long-term variability of our sources. In Section 5, we discuss our findings and in Section 6, we summarize our results and conclude. 


\section{DATA}

\subsection{Source Selection}

We define our sample as BHXB sources with more than one epoch of quasi-simultaneous (performed within a day of each other) radio, $\mathrm{X}$-ray, and infrared/optical/UV observations available, with the goal of covering the broadband spectral evolution in both the jets and the accretion flow from HS to HIMS as accurately as possible. We also require that epochs should have power-law dominated X-ray spectra and a flat or inverted radio spectrum, as well as timing characteristics consistent with the HS or HIMS. We do not consider any SIMS/SS epochs as the compact jets are not active and the corona is sub-dominant. Because the power colours are only defined for the RXTE/PCA energy band (Heil et al. 2015a), we restrict our analysis to sources which have RXTE (Jahoda et al. 1996) observations. Therefore, we do not include a few more recent BHXB sources in our search (e.g. Uttley et al. 2018; Armas Padilla et al. 2019; Shang et al. 2019; Xu et al. 2020). Calibrating the power colours across different instruments is an interesting problem to be considered in future works.

The data base used for selecting the sources in this work is the Whole-sky Alberta Time-resolved Comprehensive black-Hole Database Of the Galaxy, or WATCHDOG (Tetarenko et al. 2016). This comprehensive data base includes 47 transient and 10 persistent XRB sources with confirmed black holes or black hole candidates as the primary objects. The total number of transient outbursts recorded is more than 130 over the two decades considered in this data base (1996-2015). We then searched the literature to check which of these sources fulfils our selection criteria. Three optimal sources were identified for this work: XTE J1752-223, MAXI J1659-152, and XTE J1650-500. The full details of the observations analysed can be found in the Appendix.

\subsection{Data reduction and collection}

We complement our RXTE hard X-ray data with soft X-ray data from the XRT instrument on-board Neil Gehrels Swift satellite, and optical or UV data from Swift/UVOT. X-ray spectra from Swift/XRT are extracted using the online xrtpipeline provided by Evans et al. $(2009)^{1}$; we only consider the $1.0-10 \mathrm{keV}$ energy range in order to avoid detector features below $1 \mathrm{keV}$. Spectra are grouped to reach a minimum signal-to-noise ratio of 20 before spectral analysis. We include any available Swift/UVOT observations taken together with the Swift/XRT data. All the optical/UV images of Swift/UVOT, if available, are taken directly from the archive, and uvotsource is used to extract the photometry of selected sources from the image. The source extraction regions are chosen to be circular, with the centres the same as the coordinates of our sources provided in the SIMBAD astronomy data base (Wenger et al. 2000), ${ }^{2}$ using a radius of 5 arcsec as suggested by the Swift team. ${ }^{3}$ The backgrounds are chosen to be a larger circular area without any other sources and close to our targets, in order to minimize the impact of background fluctuations.

Radio, IR, and other optical/UV data in this work are taken from the literature on each source [for XTE J1650-500: Corbel et al. (2004), Curran, Chaty \& Heras (2012); XTE J1752-223: Brocksopp et al. (2013); MAXI J1659-152: van der Horst et al. (2013)]. We perform the de-reddening of IR/optical/UV data ourselves to make sure the

\footnotetext{
${ }^{1}$ https://www.swift.ac.uk/user_objects/

${ }^{2}$ http://simbad.u-strasbg.fr/simbad/

${ }^{3}$ https://www.swift.ac.uk/analysis/uvot/mag.php
}

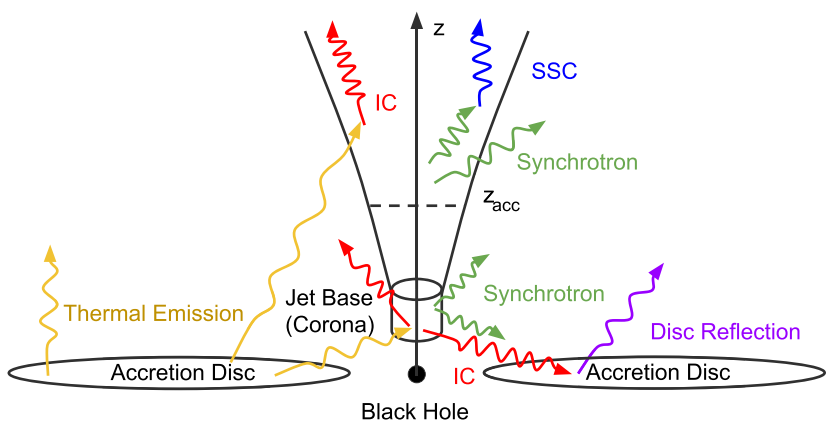

Figure 2. Schematic of the emission model used in this work. The thermal photons from the accretion disc are IC up-scattered by the synchrotronproducing electrons inside the jet; the jet cyclo-synchrotron emission is also subject to self-Comptonization (SSC). The jet base serves as the effective corona to re-illuminate the disc and generate the disc reflection spectrum, calculated by model reflect (Magdziarz \& Zdziarski 1995). Jet model parameters are listed in Table 1.

observations are consistently treated. The hydrogen column density $N_{H}$ in $\mathrm{cm}^{-2}$ related to the Galactic extinction for each target is adopted from existing publications. We estimate the extinction using the $A_{V}$ $\sim N_{H}$ relation in Foight et al. (2016): $N_{H}=(2.87 \pm 0.12) \times 10^{21} A_{V}$. Finally, we re-scale the extinction $A_{V}$ to various IR/optical/UV bands $A_{\omega}$ using the online tool ${ }^{4}$ based on the extinction law studied in Cardelli, Clayton \& Mathis (1989). The measured IR/optical/UV flux in $\mathrm{mJy}$ at wavelength $\omega$ band is de-reddened by a factor of $10^{0.4 A_{\omega}}$. This allows us to use consistent extinction/absorption in the IR/optical/UV and the X-ray bands, i.e. the $N_{H}$ in the X-ray extinction model TBabs (Wilms, Allen \& McCray 2000) during spectral fits.

\section{MULTI-WAVELENGTH JET MODEL}

Bljet, the spectral model used in this work to fit the timeindependent, broadband spectra (radio through X-rays or $\gamma$-rays) of accreting black holes is an update of the model presented first in Lucchini et al. (2019), and further developed in Lucchini et al. (2021). Briefly, bljet is a steady-state, multi-zone, semi-analytical model, designed to qualitatively mimic the dynamical jet profiles observed in GRMHD simulations, while being computationally inexpensive enough to allow complex multi-wavelength data analysis. The previous version (called agnj et) has mainly been used in studies of hard states and the quiescence of BHXBs (e.g. Markoff et al. 2005; Gallo et al. 2007; Connors et al. 2017). It can also reproduce the broadband SEDs of several low luminosity AGN (e.g. Markoff et al. 2001b, 2008; Maitra et al. 2011; Markoff et al. 2015; Connors et al. 2017). The details of the jet dynamics can be found in Lucchini et al. (2019), while the treatment of the particle distributions and radiative mechanisms are described in Lucchini et al. (2021).

Fig. 2 gives a schematic of the model geometry used in this work. Besides a truncated Shakura-Sunyaev accretion disc (Shakura \& Sunyaev 1973, 1976), the model parametrizes the power injected at the base of the jet as a percentage $N_{j}$ of the black hole's Eddington luminosity $L_{\text {Edd }}$, divided among hot electrons, cold protons, and magnetic fields. We only consider leptonic radiative processes (but see also Kantzas et al. 2021 for a version of the model which includes the contribution of hadronic processes); the protons carried in the jet are always assumed to be cold and therefore do not contribute to the

\footnotetext{
${ }^{4}$ http://www.dougwelch.org/Acurve.html
} 
Table 1. A full list of parameters and descriptions in the jet model used in this work. Parameters in bold font are left free during spectral fitting, while the others are frozen.

\begin{tabular}{ll}
\hline Parameter & \multicolumn{1}{c}{ Description } \\
\hline$N_{j}$ & Total power channeled into the jet base, in units of Eddington luminosity $L_{\mathrm{Edd}}$. \\
$\boldsymbol{R}_{\mathbf{0}}$ & Radius of the cylindrical jet base/corona in units of $\mathrm{R}_{\mathrm{g}}$. \\
$\boldsymbol{T}_{\boldsymbol{e}}$ & Temperature of the thermal electrons injected in the corona in units of keV. \\
$f_{\mathbf{p l}}$ & Free parameter used to model inverted, rather than flat $\left(f_{\mathrm{pl}}=0\right.$ ), radio spectra. \\
$\boldsymbol{R}_{\mathrm{in}}$ & Radius of the inner edge of the thin accretion disc in units of $\mathrm{R}_{\mathrm{g} .}$ \\
$\boldsymbol{L}_{\mathbf{d i s c}}$ & Luminosity of the thin disc in units of $L_{\mathrm{Edd}}$. \\
$M_{\mathrm{BH}}, \theta_{i}, d$ & Mass, inclination angle, and distance of the black hole. We fix these parameters to reported values in the literature. See Table 3 in results. \\
$z_{\mathrm{acc}}=2000 \mathrm{R}_{\mathrm{g}}$ & Distance along the jet where the jet bulk acceleration ends and particle acceleration initiates. \\
$\epsilon_{p l}=0.1$ & The fraction of initially thermal particles accelerated into a power-law tail at $z_{\text {acc. }}$. \\
$p=2.3$ & Slope of the power law of the non-thermal particle distribution. \\
$f_{b}=0.1$ & Adiabatic cooling efficiency. It sets the location of the cooling break in the radiating particle distribution. \\
$\beta_{p}=0.0315$ & Standard plasma- $\beta$ parameter at the base of the jet. A given set of $\gamma_{\text {acc }}$ and $\sigma_{\mathrm{f}}$ determines the number ratio between protons and \\
$f_{\mathrm{sc}}=0.1$ & electron-positron pairs. Our choice corresponds to roughly $\sim 30$ pairs per proton. \\
$\sigma_{\mathrm{f}}=0.1$ & Efficiency parameter for particle acceleration, which sets the maximum energy cutoff of the particle power-law distribution. \\
$\gamma_{\mathrm{acc}}=3$ & The final magnetization parameter after the jet bulk acceleration stops at $z_{\text {acc. }}$. \\
\hline
\end{tabular}

SED. The base of the jets is a cylinder of radius $R_{0}$ and height $H=2 R_{0}$ located above the accretion disc and at an initial height of $Z_{0}=2 \mathrm{R}_{\mathrm{g}}$ above the disc and black hole (although note that the model currently does not include general relativistic effects, and therefore we never fit for $Z_{0}$ ). For typical parameters, the emission from the base of the jets resembles that of a typical black hole corona, and therefore our model can be thought of as a physical realization of the lamp-post model. Hereafter the terms 'corona' and 'jet base' are used interchangeably. We include the thermal cyclo-synchrotron emission from near the base of the jet, the non-thermal synchrotron emission downstream in the jet, and the inverse-Compton (IC) scattering of both disc thermal photons and cyclo-synchrotron photons. The coronal emission then re-illuminates the accretion disc and generates the disc reflection spectrum (e.g. Markoff \& Nowak 2004; Fabian \& Ross 2010); in this work, we treat reflection using the phenomenological model reflect (Magdziarz \& Zdziarski 1995). Additionally, bljet can also include an optical blackbody excess to mimic the optical/UV emission from the disc irradiation (e.g. Gierliński, Done \& Page 2009).

Details of the jet acceleration and collimation profile are discussed in Lucchini et al. (2019). The jet bulk acceleration begins at the top of the corona, effectively converting the initial magnetic field content of the jet into bulk kinetic energy up to a distance $z_{\text {acc }}$ from the black hole. At a distance $z_{\text {diss }}$, which we take to be equal to $z_{\text {acc }}$ for simplicity, the jet experiences a dissipation region, where a fraction $\epsilon_{p l}=10$ per cent of the thermal electrons are channelled into a nonthermal, power-law particle distribution tail with an index of $p$. This dissipation region represents the start of particle acceleration in the jet (Markoff et al. 2005) which sets the start of the flat/inverted spectrum (Blandford \& Königl 1979; Boettcher \& Dermer 2010; Malzac 2013), possibly due to internal shocks driven by fluctuations in the outflow velocity (Malzac 2014), or instabilities along the jet boundary (Chatterjee et al. 2019). As the plasma moves downstream of $z_{\text {acc }}$, the percentage of accelerated particles along the jet is reduced by a factor $\left(\log _{10}\left(z_{\mathrm{acc}}\right) / \log _{10}(z)\right)^{f_{\mathrm{pl}}}$. The parameter $f_{\mathrm{pl}}$ allows us to artificially suppress the cyclo-synchrotron emissivity from successive jet regions outwards along the jets, resulting in an inverted, rather than flat, radio spectrum. This parameter in effect fudges the complex balance between particle cooling and re-acceleration that we plan to explore in future work. A list of the jet parameters and descriptions concerning this work are listed in Table 1.
We freeze several parameters in our SED modelling in order to reduce model degeneracy. BHXB jets are likely only moderately relativistic (Fender et al. 2004), and hence we take the terminal bulk Lorenz factor of the jet to be $\Gamma=3$. The final magnetization parameter $\sigma_{\mathrm{f}}$ after the bulk acceleration at $z_{\text {acc }}$ is set to be 0.1 , as in Lucchini et al. (2021). In our data, the optically thin part of the non-thermal synchrotron spectrum is not well sampled, and therefore we freeze $p$ and $z_{\text {acc }}$ to 2.3 and $2000 \mathrm{R}_{\mathrm{g}}$, respectively, in agreement with the estimates by Gandhi et al. (2008) for the canonical BHXB GX 339-4. This choice of $p$ and $z_{\text {acc }}$ causes the X-ray flux of the non-thermal synchrotron to fall well below the IC emission from the jet base. The standard plasma parameter $\beta_{p}=U_{e} / U_{B}$ at the base of the jet is set to 0.0315 . This choice of the $\beta_{p}$, together with the final Lorenz factor $\gamma_{\mathrm{acc}}$ and the final magnetization $\sigma_{\mathrm{f}}$, sets the number ratio between electron-positron pairs and protons to be $\approx 30$, resulting in jet powers that are roughly on the order of the accretion disc luminosity, similarly to Lucchini et al. (2021). A pair-dominated region near the black hole would be a natural consequence of several mechanisms for pair-loading in the black hole a magnetosphere (e.g. Neronov \& Aharonian 2007; Mościbrodzka et al. 2011; Broderick \& Tchekhovskoy 2015). Other parameter values fixed among fits can be found in Table 1 as well.

The two primary geometrical parameters we focus on in this work are the size of the jet base $R_{0}$, and the radius of the inner edge of the Shakura-Sunyaev disc $R_{\text {in }}$. Crucially, $R_{0}$ scales the energy densities and optical depth of the corona, and thus has a large impact on the flux of the thermal synchrotron emission inside the corona, as well as on the normalization and slope of the IC spectrum.

\section{RESULTS}

\subsection{Overall spectral and timing behaviour}

Fig. 3 shows the HIDs and the PCD of outbursts from our selected BHXBs: XTE J1752-223, MAXI J1659-152, and XTE J1650-500. The X-ray hard colour in the HIDs is calculated as the ratio of source counts between $8.6-18.0 \mathrm{keV}$ and $5.0-8.6 \mathrm{keV}$. All epochs with quasi-simultaneous observations are labelled with letters in the HIDs and are shown in the PCD. To illustrate the chronology of the selected multi-wavelength epochs, we use the HID to map the full outburst of 


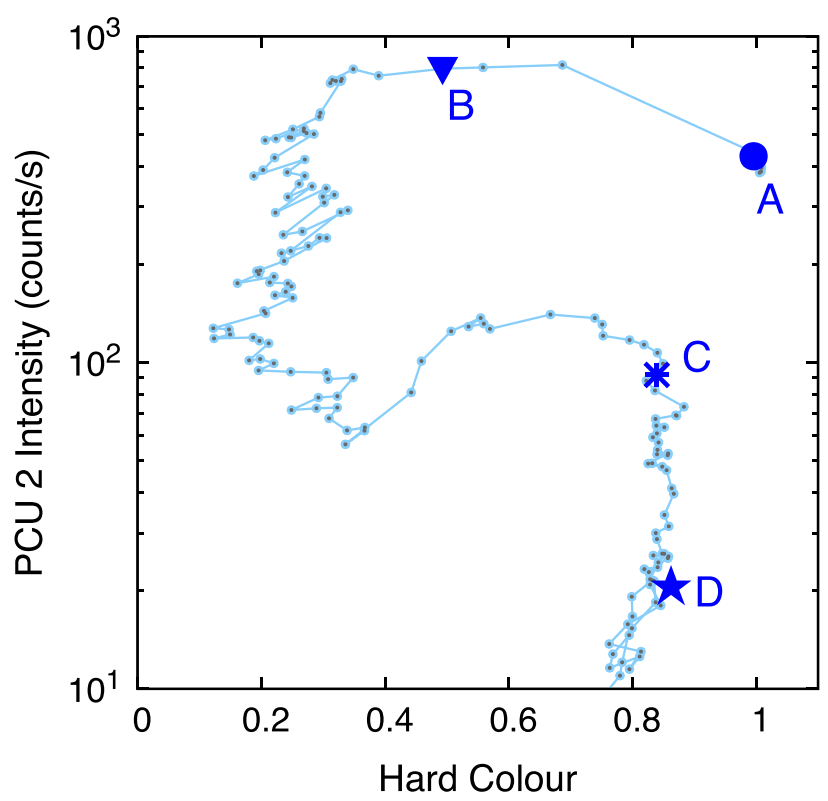

(a)

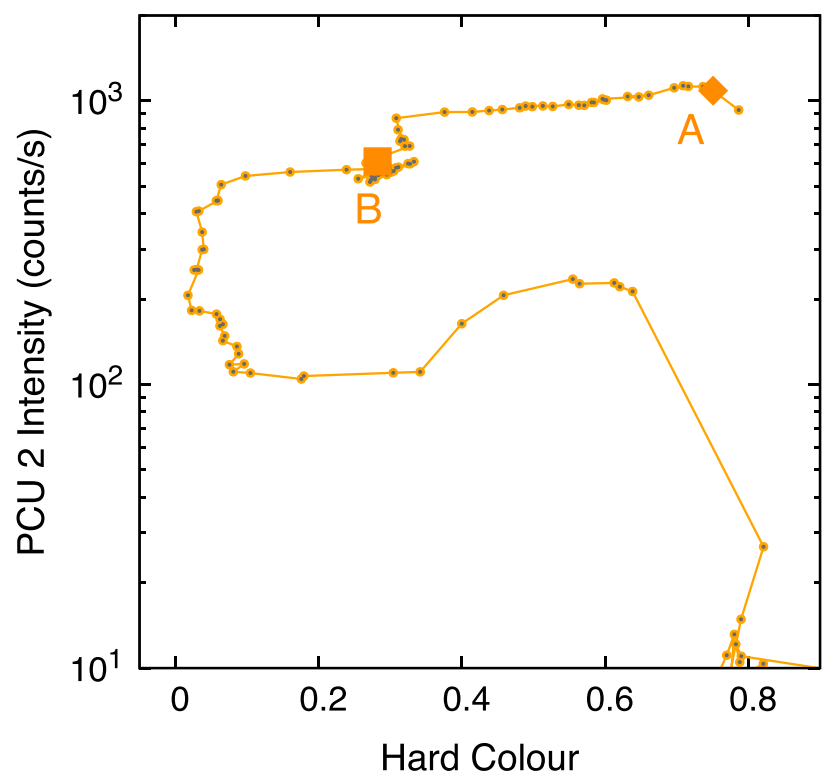

(c)

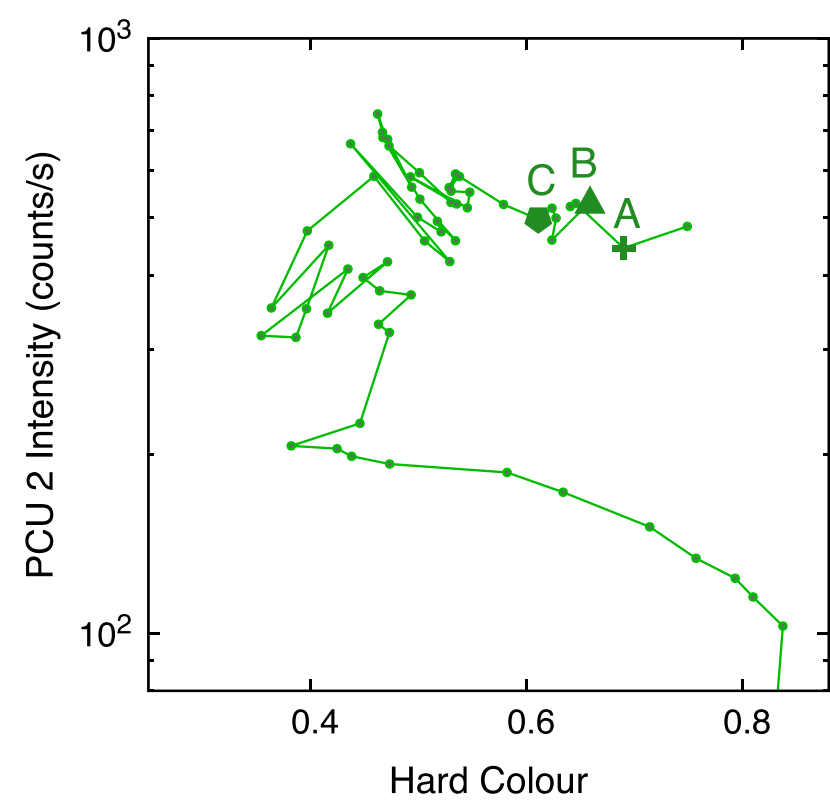

(b)

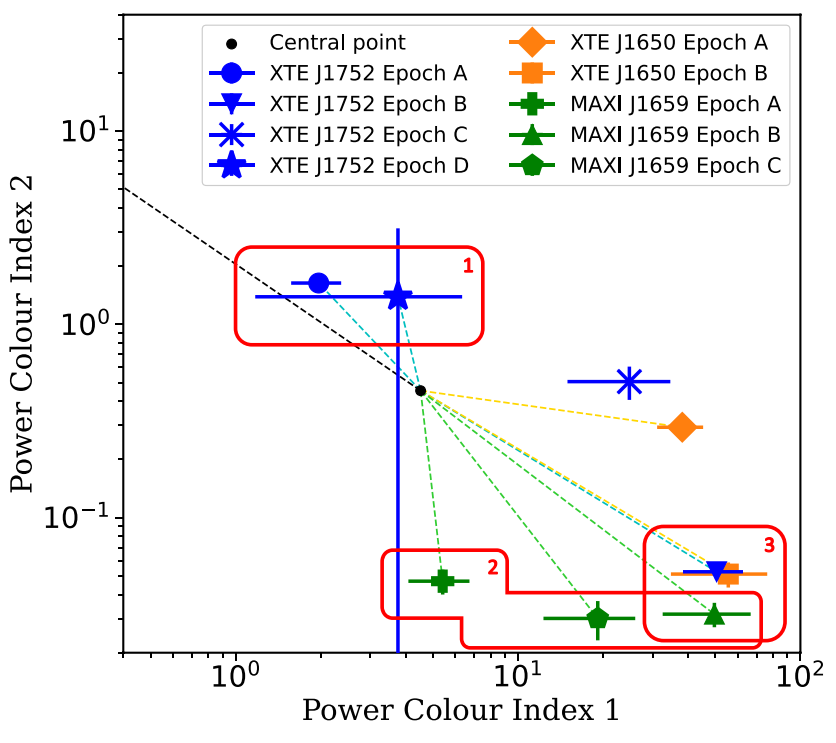

(d)



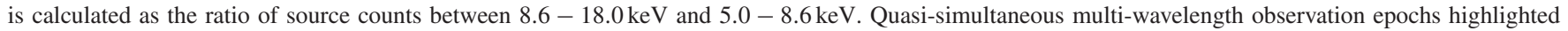

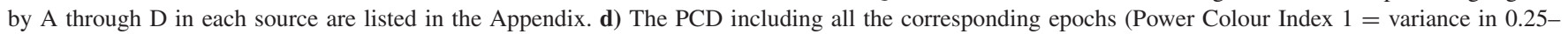

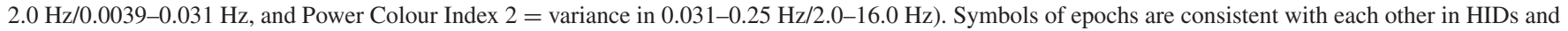

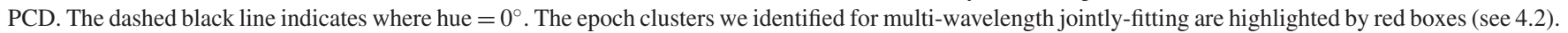

each source, and the data reduction to produce the HIDs is identical to that used in Connors et al. (2020).

We identify three epoch clusters of interests from the PCD, and focus our spectral analyses of these clusters by performing joint spectral fits of the broadband SEDs for each (Fig. 3d): Cluster 1 consists of XTE J1752-223 epochs A\&D, which have similar hues; these are from a bright hard state during the rise of the outburst, and a faint hard state during the decay, respectively. Cluster
2 consists of MAXI J1659-152's Epochs A\&B\&C, capturing the gradual changes in spectral and timing properties during the hard-tosoft state transition of the source. Cluster 3 consists of bright hardintermediate states from all three objects (XTE J1752-223 Epoch B, MAXI J1659-152 Epoch B, and XTE J1650-500 Epoch B), allowing us to probe states showing near identical hues but different spectral characteristics. Although XTE J1752-223 on 2010-01-21 (Epoch B) only has X-ray and radio observations, we find its power-colour hue 

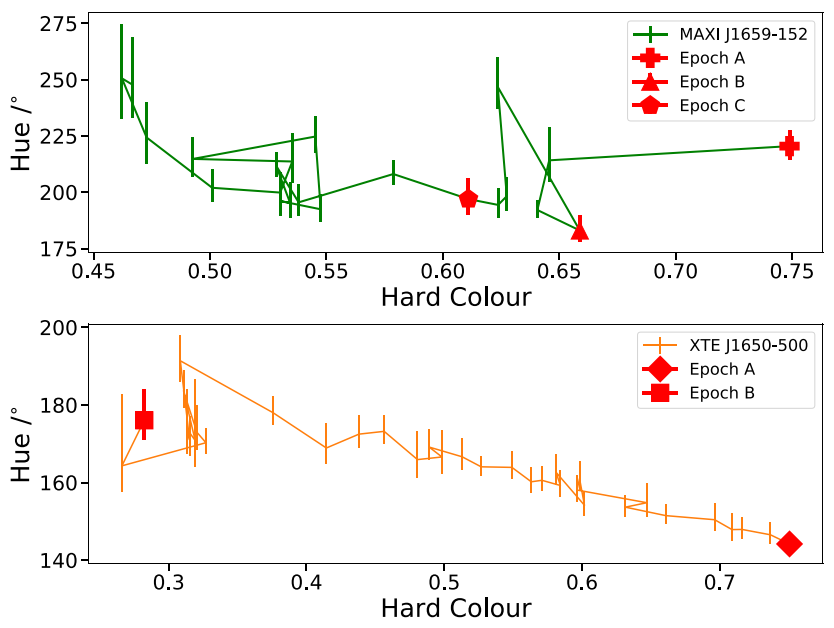

Figure 4. Evolution of the power spectral hue in MAXI J1659-152 and XTE J1650-500 with the hardness, focused on observations close to the selected epochs in this work. Epochs A through $\mathrm{C}$ are highlighted by red data points for each source. During the concerned period of outburst, the hue of MAXI J1659-152 changed rapidly, and does not appear to strongly follow the spectral changes; XTE J1650-500 showed a more prominent trend of the hue increasing as the source transited from hard states to soft states over time.

is consistent with two epochs from the other two sources (J1650B and J1659B), and so we make an exception of the selection criteria and keep this epoch in our following multi-wavelength spectral analysis in order to exploit the X-ray timing similarity across sources. On the other hand, XTE J1752-223 Epoch C and XTE J1650-500 Epoch A have hue measurements that differ from each other at the $\approx 2-3 \sigma$ level, and furthermore, they happen at different outburst stages from different sources. This makes it harder to directly compare the two, and thus we do not form an additional cluster containing these two epochs. We only proceed with the clustered epochs in the following spectral analyses.

Our findings of the power colours in three BHXB sources are mostly consistent with the previous study on 12 BHXB systems (Heil et al. 2015a): epochs with states of different spectral hardness occupy different angular regions on the PCD. We notice that the three selected MAXI J1659 epochs show a type-C Quasi-Periodic Oscillation (QPO) in their power spectra (Kalamkar et al. 2011), which combined with high inclination ( $>60^{\circ}$, also see the Appendix for a discussion on the inclination estimation of J1659) can alter the evolution track of the BHXB source on the PCD (Fig. 1) and mainly push the track to have lower PC2 values in the concerned range of hue for these three epochs (Heil, Uttley \& Klein-Wolt 2015b). However, for the purposes of our study this effect is minimal and does not affect our selection of epoch clusters. Therefore, in this work we do not remove any QPOs before calculating the hue. In addition, we find that the hue can also fluctuate in individual PCD tracks while the sources become softer in X-rays (Fig. 4), blurring the generally increasing trend of hue (moving clockwise on the PCD) at these outburst stages. These results show that some additional caution when interpreting the empirical spectral-timing correlation in individual BHXB sources should be taken.

Our next step is to perform spectral analyses of the epochs in each of the three clusters. Before applying the multi-wavelength jet model, we perform phenomenological modelling to the X-ray data in order to quantify the amount of reflection in each epoch, as this is a very important constraint on jet models: as a rule of thumb, low or absent reflection fraction and/or narrow iron $\mathrm{K}-\alpha$ lines favour non-

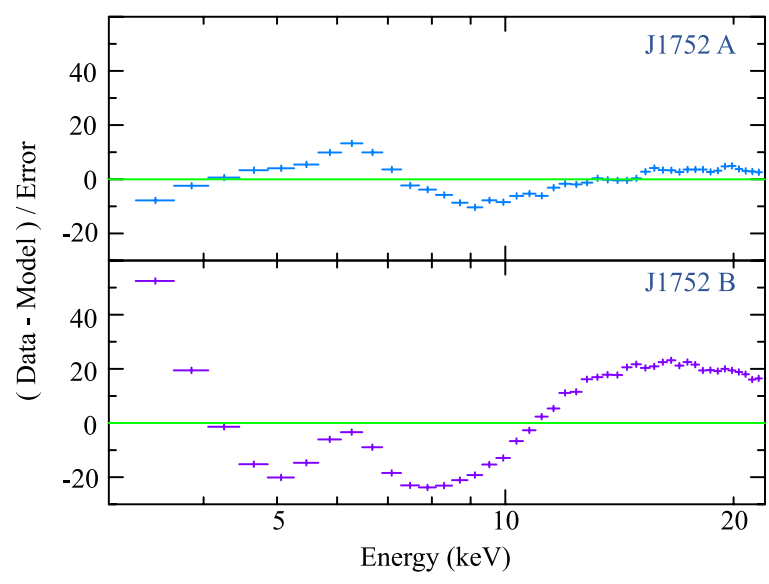

Figure 5. Residuals of XTE J1752-223 Epoch A (2009-11-05) and B (201001-21) RXTE/PCA data fitted with a power-law model. The slope and normalization of the model are different between these two epochs. Both epochs show the broad iron $\mathrm{K}-\alpha$ emission at $6.4 \mathrm{keV}$ and the Compton hump $\gtrsim 10 \mathrm{keV}$, indicating the presence of the disc reflection. Moreover, J1752B shows a powerful soft component that comes from the thermal disc emission, signaling the ongoing state transition (Fig. 3a).

thermal synchrotron emission as the origin of the X-ray power-law, while large reflection fractions and/or broad lines favour IC emission near the jet base (Markoff \& Nowak 2004). The fitting and statistical analysis of the phenomenological modelling is carried out using the XSPEC package (version 12.11.1; Arnaud 1996). By using a simple power-law model, we identify broad iron $\mathrm{K}-\alpha$ emission and the Compton hump in the fit residuals of several epochs, indicating the presence of relativistic disc reflection. Examples of the fitting residuals can be found in Fig. 5. We then use the phenomenological model TBabs*[reflect (powerlaw) +diskbb + Gaussian] to model the X-ray data of all three clusters. We use the model reflect (Magdziarz \& Zdziarski 1995) plus a Gaussian line profile fixed at $6.4 \mathrm{keV}$ to model the disc reflection features, while diskbb covers the disc thermal photons. We set the iron abundance of the disc to the solar abundance, except for XTE J1752, in which it is set to 3.5 times the solar abundance (García et al. 2018). Bestfitting parameters are summarized in Table 2. We note that for the two epochs in Cluster 1 the disc normalization is abnormally high compared to other epochs. This difference is because the disc temperature $T_{i n}$ is lower in these two epochs, making the disc component constrained only by the soft end of the Swift/XRT data. Combined with our choice to fix the absorption $N_{H}$, this makes our phenomenological fits only a rough estimation on the disc component and should be interpreted with caution. We also observe the disc component falling almost out of the X-ray band in our multiwavelength SED modelling of these two epochs (Fig. 6).

\subsection{Multi-wavelength SEDs}

We then model the broadband SEDs using bl jet to explore possible physical similarities among epochs and epoch clusters. To better constrain the model, we test these similarities by performing joint-fits for each cluster, where we model the epochs in one cluster simultaneously and search for best-fits with certain parameters tied between epochs. We use the Interactive Spectral Interpretation System (ISIS, version 1.6.2-43; Houck \& Denicola 2000) to perform our broadband spectral fits, because it allows the forward-folding of the full jet model into X-ray detector space while simultaneously fitting the radio, IR, 
Table 2. Best-fitting parameters of the selected epochs with phenomenological model TBabs*[reflect (powerlaw) +diskbb + Gaussian]. Parameters fixed to certain values are shown in brackets. Unconstrained parameters are given with their upper limits in 90 per cent confidence level. Swift/XRT and RXTE/HEXTE data are included when available. Galactic absorption for each source is fixed to reported values in the literature (XTE J1650-500:Miniutti, Fabian \& Miller 2004; MAXI J1659-152:van der Horst et al. 2013; XTE J1752-223:García et al. 2018). Epochs in the same cluster are modelled individually, and J1659B is modelled only once while it is displayed in both Cluster 2 and Cluster 3 for comparison purpose.

\begin{tabular}{|c|c|c|c|c|c|c|c|c|c|}
\hline \multirow[b]{2}{*}{ Model component } & \multirow[b]{2}{*}{ Parameter } & \multicolumn{2}{|c|}{ Cluster 1} & \multicolumn{3}{|c|}{ Cluster 2} & \multicolumn{3}{|c|}{ Cluster 3} \\
\hline & & J1752 A & J1752 D & J1659 A & J1659 B & $\mathrm{J} 1659 \mathrm{C}$ & J1752 B & J1659 B & J1650 B \\
\hline TBabs & $N_{H}\left(10^{22} \mathrm{~cm}^{-2}\right)$ & \multicolumn{2}{|c|}{$(1.0)$} & \multicolumn{3}{|c|}{$(0.32)$} & $(1.0)$ & $(0.32)$ & $(0.5)$ \\
\hline Powerlaw & $\begin{array}{c}\Gamma \\
A_{\text {powerlaw }}\end{array}$ & $\begin{array}{l}1.525_{-0.007}^{+0.007} \\
0.610_{-0.007}^{+0.011}\end{array}$ & $\begin{array}{l}1.735_{-0.002}^{+0.003} \\
0.048_{-0.002}^{+0.004}\end{array}$ & $\begin{array}{c}2.056_{-0.008}^{+0.008} \\
1.74_{-0.03}^{+0.03}\end{array}$ & $\begin{array}{l}2.17_{-0.02}^{+0.03} \\
2.41_{-0.08}^{+0.12}\end{array}$ & $\begin{array}{c}2.137_{-0.005}^{+0.025} \\
2.00_{-0.12}^{+0.01}\end{array}$ & $\begin{array}{c}2.17_{-0.07}^{+0.07} \\
2.7_{-0.4}^{+0.5}\end{array}$ & $\begin{array}{l}2.17_{-0.02}^{+0.03} \\
2.41_{-0.08}^{+0.12}\end{array}$ & $\begin{array}{l}2.4_{-0.1}^{+0.2} \\
1.1_{-0.3}^{+0.4}\end{array}$ \\
\hline Diskbb & $\begin{array}{c}T_{\text {in }}(\mathrm{keV}) \\
A_{\text {diskbb }}\end{array}$ & $\begin{array}{c}0.28_{-0.03}^{+0.03} \\
1.4_{-0.6}^{+1.3} \times 10^{4}\end{array}$ & $\begin{array}{c}0.16_{-0.04}^{+0.04} \\
5_{-4}^{+79} \times 10^{4}\end{array}$ & $\begin{array}{c}0.52_{-0.02}^{+0.02} \\
1.1_{-0.2}^{+0.2} \times 10^{3}\end{array}$ & $\begin{array}{c}0.61_{-0.02}^{+0.02} \\
7.6_{-0.9}^{+1.1} \times 10^{2}\end{array}$ & $\begin{array}{c}0.772_{-0.005}^{+0.016} \\
4.0_{-0.1}^{+0.5} \times 10^{2}\end{array}$ & $\begin{array}{c}0.76_{-0.03}^{+0.02} \\
2.0_{-0.3}^{+0.3} \times 10^{3}\end{array}$ & $\begin{array}{c}0.61_{-0.02}^{+0.02} \\
7.6_{-0.9}^{+1.1} \times 10^{2}\end{array}$ & $\begin{array}{c}0.661_{-0.008}^{+0.008} \\
7.1_{-0.5}^{+0.5} \times 10^{3}\end{array}$ \\
\hline Gaussian & $\begin{array}{c}E_{\text {center }}(\mathrm{keV}) \\
\sigma_{\text {gauss }} \\
A_{\text {gauss }}\end{array}$ & $\begin{array}{c}<0.6 \\
5_{-4}^{+4} \times 10^{-4}\end{array}$ & $\begin{array}{l}\text { 4) } \begin{array}{c}<1.1 \\
3_{-2}^{+2} \times 10^{-4}\end{array}\end{array}$ & $\begin{array}{c}<0.01 \\
9_{-4}^{+4} \times 10^{-4}\end{array}$ & $\begin{array}{c}(6.4) \\
<0.6 \\
10_{-5}^{+5} \times 10^{-4}\end{array}$ & $\begin{array}{c}1.1_{-0.3}^{+0.4} \\
0.004_{-0.001}^{+0.001}\end{array}$ & $\begin{array}{c}0.91_{-0.07}^{+0.08} \\
0.020_{-0.003}^{+0.003}\end{array}$ & $\begin{array}{c}(6.4) \\
<0.6 \\
10_{-5}^{+5} \times 10^{-4}\end{array}$ & $\begin{array}{c}1.10_{-0.02}^{+0.02} \\
0.015_{-0.001}^{+0.001}\end{array}$ \\
\hline$\chi^{2} /$ d.o.f & & 277/174 & $35 / 53$ & 195/120 & $304 / 179$ & $159 / 100$ & $79 / 38$ & $304 / 179$ & $74 / 51$ \\
\hline
\end{tabular}

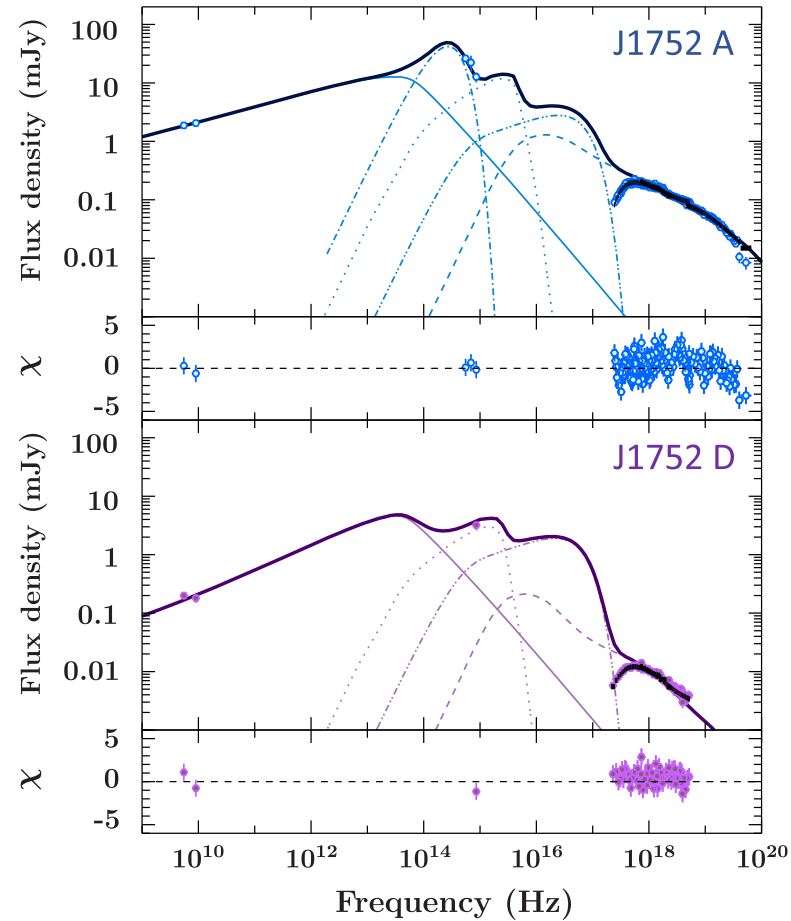

Figure 6. Best joint-fit of the two epochs in Cluster 1. The thick continuous dark line shows the total model, the thin continuous line shows the nonthermal synchrotron emission, the thin dashed line shows the IC emission from the jet base, the thin dotted line shows the thermal cyclo-synchrotron emission from the jet base, the dot-dashed and double dot-dashed lines show the optical blackbody excess and accretion disc, the triple dot-dashed line represents reflection.

optical, and UV data. We explore the parameter space of our model by using the emcee Markov chain Monte Carlo algorithm (ForemanMackey et al. 2013) implementation in ISIS. We run the emcee for a total of 5000 steps and discard the first 2000 steps, which in all of our cases ensures the convergence of the chain. We use 20 walkers per free parameter for each chain and we define the value of the best-fitting parameter as the median of the walker distribution after the chain burn-in period. We define the $1 \sigma$ uncertainty as the interval
Table 3. Fixed parameters of the sources in this work. $M_{\mathrm{BH}}$ is the black hole mass in units of $M_{\odot}, d$, and $\theta_{i}$ the distance and inclination angle of the system, and $N_{H}$ the hydrogen column density for interstellar extinction. We freeze all of these values in our spectral fits.

\begin{tabular}{lccccc}
\hline & $M_{\mathrm{BH}}\left(M_{\odot}\right)$ & $d(k p c)$ & $\theta_{i}\left({ }^{\circ}\right)$ & $N_{H}\left(10^{22} \mathrm{~cm}^{-2}\right)$ & References \\
\hline XTE J1752-223 & 9.6 & 6 & 35 & 1.0 & $1,2,3$ \\
MAXI J1659-152 & 6 & 6 & 75 & 0.32 & $4,5,6,7$ \\
XTE J1650-500 & 5.1 & 2.6 & 45 & 0.5 & $8,9,10$
\end{tabular}

Note.1: Shaposhnikov et al. (2010); 2: García et al. (2018); 3: Ratti et al. (2012); 4: Molla et al. (2016); 5: Kuulkers et al. (2013); 6: Kong (2012); 7: van der Horst et al. (2013); 8: Slanỳ \& Stuchlik (2008); 9: Miniutti et al. (2004); 10: Homan et al. (2006).

in the posterior distribution which contains 68 per cent of the walkers after excluding the burn-in period.

We fix the mass $\boldsymbol{M}_{\mathbf{B H}}$, inclination angle $\boldsymbol{\theta}_{\boldsymbol{i}}$, and distance $\boldsymbol{d}$ of the black hole to values reported in the literature, summarized in Table 3. The syntax of the multi-wavelength model is 'constant*TBabs*(reflect*bljet + Gaussian)', where the calibration factors between different instruments are included via the constant model within ISIS. Following Lucchini et al. (2021), we try to tie the geometric parameters of the X-ray emitting region $\left(R_{0}\right.$ and $\left.R_{\text {in }}\right)$ in each of our epoch clusters when running our joint-fits. The best-fitting results are summarized in Figs $6,7,8$, and Table 4 . We could not find any satisfactory fits with both $R_{0}$ and $R_{\text {in }}$ tied in Cluster 2 or Cluster 3 , and instead we find good fits with either $R_{0}$ (Cluster 2$)$ or $R_{\text {in }}$ (Cluster 3 ) tied in these two joint-fits. Because J1659B is shared by Cluster 2 and Cluster 3 , we model this epoch twice in the joint-fits of these two cluster. While the best-fitting parameters of J1659B in these two joint-fits are only marginally consistent with each other, this minor discrepancy does not impact the evolution of $R_{0}$ or Refl $\mathrm{frac}_{\text {fra }}$, we infer from the fits (see Section 5). We also check our approach of tying $R_{0}$ and/or $R_{\text {in }}$ parameters by re-doing the joint-fits but with the $R_{0}$ and $R_{\text {in }}$ untied among all the epochs. We find that $R_{0}$ changes less than $1 \sigma$ in most epochs (except for J1659B in Cluster 2 it is less than $3 \sigma$ ), while $R_{\text {in }}$ changes $\sim 20$ percent in Cluster 1 and Cluster 2 (see Table A9 in the Appendix). We discuss various sources of the $R_{\text {in }}$ uncertainty in our analyses in the next section.

In all epochs, our model is in good agreement with the broadband SEDs. None of the epochs has sufficient data to constrain the location 


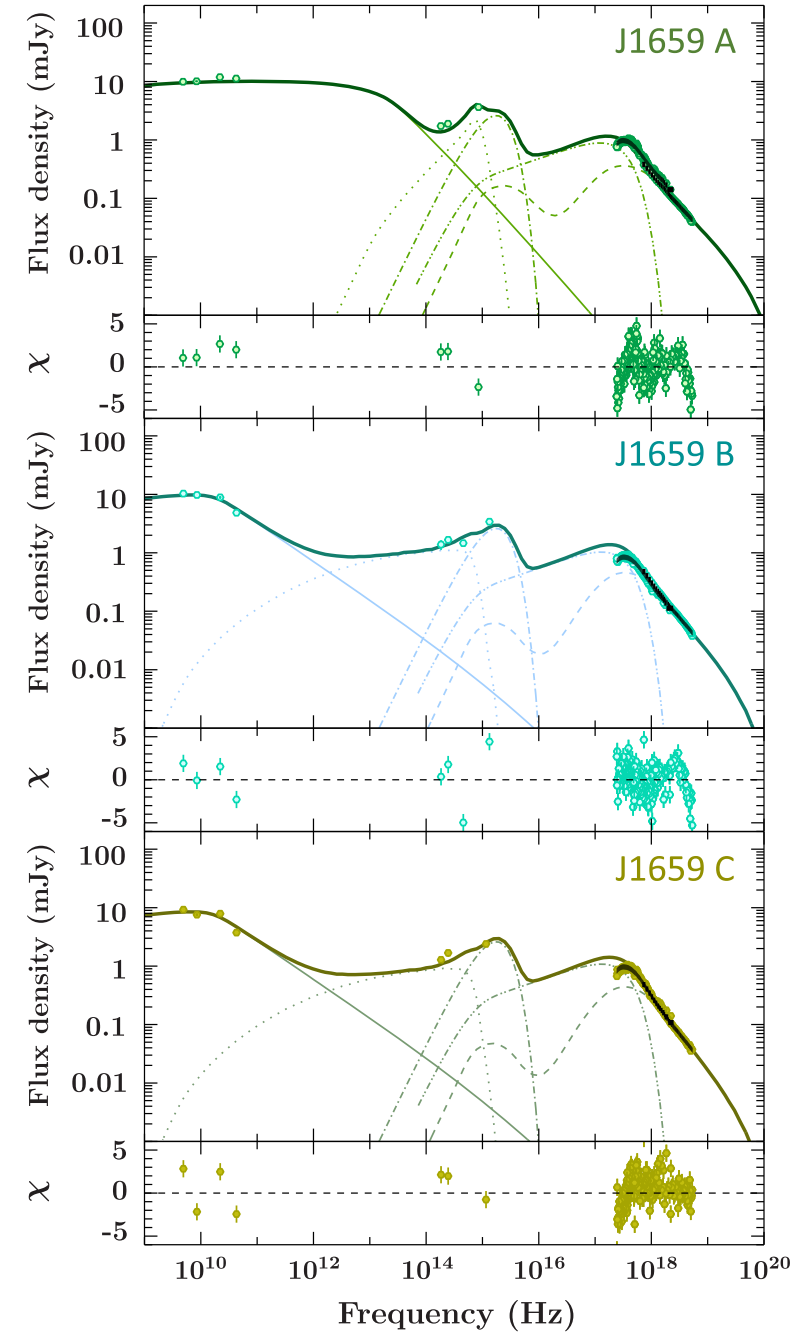

Figure 7. Best joint-fit of SEDs in Cluster 2. Same format as Fig. 6.

of the jet spectral break (e.g. Corbel \& Fender 2002; Russell et al. 2013), while for MAXI J1659-152 the break is only indicated by four radio bands in two epochs (J1659 B\&C), indicating that the particle acceleration region is very far from the black hole (Russell et al. 2013). The dominant mechanism responsible for the optical to $\mathrm{UV}$ band in J1752D and J1650B is the thermal synchrotron emission in the jet base, while four other epochs (J1752A and J1659A/B/C) display an excess in flux (dominant in J1752A) which cannot be fully covered by the coronal synchrotron emission. We model the excess by an additional blackbody component. One of the possible explanation for this thermal excess is irradiation of the outer disc from the inner X-ray emission (e.g. Maitra et al. 2009; Gierliński et al. 2009; van der Horst et al. 2013). The dominant mechanism for the X-ray emission in our model is inverse-Comptonization of jet synchrotron photons and disc photons. The up-scattered photons seeded from the disc blackbody emission dominates the hard X-ray flux except for J1752 Epoch A and Epoch D. Synchrotron-self-Comptonization (SSC) in the jet dominates the X-ray spectra in the latter two epochs (Fig. 6).

\section{DISCUSSION}

The main results of our joint-fits are as follows: in Cluster 1, our jointfit models the broadband differences between the high/low HS of

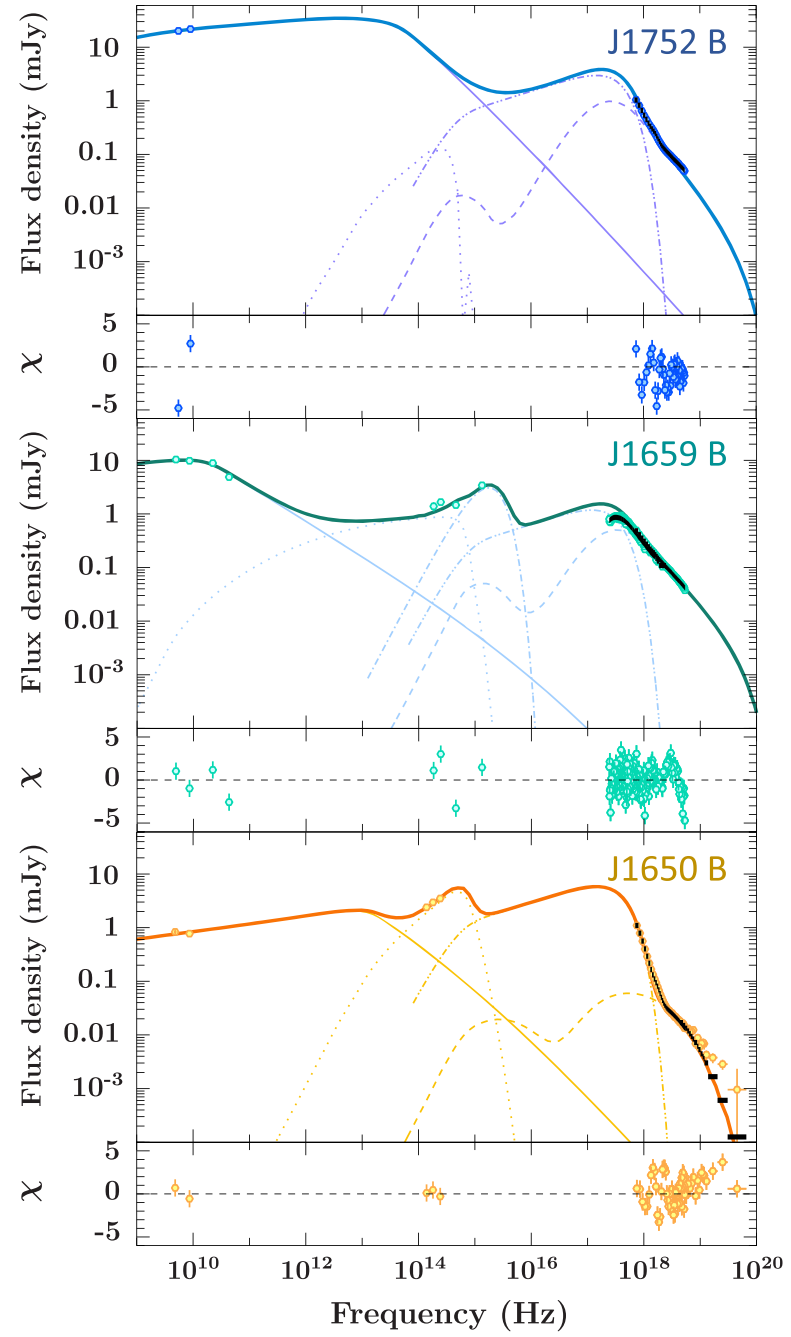

Figure 8. Best joint-fit of SEDs in Cluster 3. Same format as Fig. 6, with $\mathrm{y}$-axis range changed to cover the X-ray high-energy tail of J1650B.

J1752 indicating a change in the jet while the accretion disc remains essentially the same. We find that the drop in total luminosity between J1752A and J1752D is caused primarily by three factors: a decrease in the jet power, a decrease in the temperature of the electrons, and an increase in the $f_{\text {pl }}$ parameter (implying less efficient particle reacceleration throughout the jet). Additionally, our results suggest that at the bright hard state the disc is truncated (at $R_{\text {in }}=140_{-16}^{+13} R_{g}$ ) as it is in the faint HS with similar hardness ratio (Fig. 3a). On the other hand, García et al. (2018) finds a $R_{\text {in }}=1.7 \pm 0.4 R_{g}$ with detailed reflection spectroscopy using the reflection model relxill Cp (Dauser et al. 2014) and all of the long-stable high HS data, including the epoch we label J1752A here (but see Zdziarski et al. 2021 for a truncation radius of $R_{\text {in }} \gtrsim 100 R_{g}$ estimated at the same HS, with which our results are more consistent). An important caveat of the truncation radii estimated in this work is that the lack of UV coverage leads to the poor constraint of the disc thermal continuum. Instead, our estimates rely on matching the normalization of the Comptonized continuum, which requires computing the energy density of the disc photons as seen by the corona: the IC luminosity scales as: $L_{\mathrm{IC}} \propto$ $U_{\text {disc }} \propto L_{\text {disc }} / R_{\text {in }}^{2}$, and therefore we can constrain $R_{\text {in }}$ only indirectly. Additionally, part of the power-law continuum is due to synchrotronself Compton, rather than thermal Comptonization of disc photons. 
Table 4. Best-fitting parameters of the joint-fits in this work. Fixed parameters are shown in brackets. Unconstrained parameters are given with their upper limits in 90 per cent confidence level. Parameters tied to each other within each cluster of epochs are indicated via a shared column. All epochs use a fixed value of $2000 R_{g}$ as $z_{a c c}$ except MAXI J1659 Epoch B\&C have a fixed $z_{a c c}$ of $4 \times 10^{6} R_{g}$ in order to model the radio spectral break observed in these two epochs. Because of the lack of UV data in each of the J1659 epochs, we combine three epochs in Cluster 2 and model the UV excess phenomenologically using one blackbody component with a fixed temperature (30 000K). Both Cluster 2 and Cluster 3 have epoch J1659B thus we include this epoch in the joint-fits respectively.

\begin{tabular}{|c|c|c|c|c|c|c|c|c|c|}
\hline \multirow{3}{*}{$\begin{array}{l}\text { Model component } \\
\text { TBabs }\end{array}$} & \multirow[b]{2}{*}{ Parameter } & \multicolumn{2}{|c|}{ Cluster 1} & \multicolumn{3}{|c|}{ Cluster 2} & \multicolumn{3}{|c|}{ Cluster 3} \\
\hline & & J1752 A & $\mathrm{J} 1752 \mathrm{D}$ & J1659 A & $\mathrm{J} 1659 \mathrm{~B}$ & $\mathrm{~J} 1659 \mathrm{C}$ & $\mathrm{J} 1752 \mathrm{~B}$ & $\mathrm{~J} 1659 \mathrm{~B}$ & $\mathrm{~J} 1650 \mathrm{~B}$ \\
\hline & $N_{H}\left(10^{22} \mathrm{~cm}^{-2}\right)$ & \multicolumn{2}{|c|}{$(1.0)$} & & $(0.32)$ & & $(1.0)$ & $(0.32)$ & $(0.5)$ \\
\hline \multirow[t]{6}{*}{ Bljet } & $f_{\mathrm{pl}}$ & $8.6_{-0.4}^{+0.2}$ & $15_{-1}^{+1}$ & & (0) & & $2.94_{-0.17}^{+0.04}$ & (0) & $3.3_{-0.2}^{+0.3}$ \\
\hline & $N_{j}$ & $0.024_{-0.002}^{+0.001}$ & $0.014_{-0.002}^{+0.001}$ & $0.0395_{-0.0006}^{+0.0008}$ & $0.0324_{-0.0006}^{+0.0007}$ & $0.0294_{-0.0004}^{+0.0006}$ & $0.0374_{-0.0015}^{+0.0005}$ & $0.0335_{-0.0006}^{+0.0006}$ & $0.00316_{-0.00007}^{+0.00007}$ \\
\hline & $R_{0}\left(R_{g}\right)$ & \multicolumn{2}{|c|}{$12.3_{-0.9}^{+0.5}$} & $40.5_{-0.7}^{+0.7}$ & $33.0_{-0.4}^{+0.4}$ & $32.6_{-0.6}^{+0.6004}$ & & $34.6_{-0.5}^{+0.4000}$ & \\
\hline & $T_{e}(\mathrm{keV})$ & $242_{-7}^{+12}$ & $188_{-17}^{+18}$ & $159_{-4}^{+3}$ & $110_{-4}^{+3}$ & $107_{-4}^{+3}$ & $44_{-2}^{+2}$ & $100_{-4}^{+3}$ & $324_{-5}^{+5}$ \\
\hline & $R_{\text {in }}\left(R_{g}\right)$ & \multicolumn{2}{|c|}{$140_{-16}^{+13}$} & & $20.0_{-0.2}^{+0.2}$ & & $9.9_{-0.3}^{+0.6}$ & $24.0_{-0.4}^{+0.4}$ & $12.3_{-0.1}^{+0.2}$ \\
\hline & $L_{d i s c}\left(L_{E d d}\right)$ & $0.011_{-0.003}^{+0.002}$ & $0.007_{-0.001}^{+0.001}$ & $0.087_{-0.001}^{+0.001}$ & $0.107_{-0.002}^{+0.001}$ & $0.114_{-0.002}^{+0.001}$ & $0.069_{-0.001}^{+0.002}$ & $0.113_{-0.002}^{+0.002}$ & $0.0546_{-0.0006}^{+0.0006}$ \\
\hline \multirow{4}{*}{$\begin{array}{l}\text { Reflect } \\
\text { Gaussian }\end{array}$} & $\operatorname{Refl}_{\text {frac }}$ & $0.29_{-0.02}^{+0.02}$ & $0.5_{-0.2}^{+0.2}$ & $<5 \times 10^{-4}$ & $<4 \times 10^{-4}$ & $<3 \times 10^{-4}$ & $0.41_{-0.05}^{+0.06}$ & $0.03_{-0.02}^{+0.03}$ & $<8 \times 10^{-4}$ \\
\hline & $E_{\text {center }}(\mathrm{keV})$ & \multicolumn{2}{|c|}{$(6.4)$} & & (6.4) & & & $(6.4)$ & \\
\hline & $\sigma_{\text {gauss }}$ & $0.2_{-0.2}^{+0.2}$ & $0.004_{-0.003}^{+0.014}$ & $0.7_{-0.1}^{+0.2}$ & $0.6_{-0.1}^{+0.2}$ & $0.4_{-0.3}^{+0.4}$ & $0.85_{-0.04}^{+0.04}$ & $0.15_{-0.10}^{+0.19}$ & $1.10_{-0.02}^{+0.02}$ \\
\hline & $A_{\text {gauss }}$ & $9_{-3}^{+2} \times 10^{-4}$ & $1.7_{-0.8}^{+0.9} \times 10^{-4}$ & $3.1_{-0.5}^{+0.5} \times 10^{-3}$ & $3.1_{-0.5}^{+0.5} \times 10^{-3}$ & $1.4_{-0.4}^{+0.6} \times 10^{-3}$ & $18.4_{-0.8}^{+0.8} \times 10^{-3}$ & $1.3_{-0.2}^{+0.3} \times 10^{-3}$ & $2.00_{-0.05}^{+0.06} \times 10^{-2}$ \\
\hline \multirow[t]{2}{*}{ Black body } & $T_{b b o d y}(K)$ & $4.6_{-0.8}^{+1.0} \times 10^{3}$ & - & & $(30000)$ & & - & (30000) & - \\
\hline & $L_{b b o d y}(\mathrm{erg} / \mathrm{s})$ & $2.5_{-0.6}^{+1.5} \times 10^{36}$ & - & & $1.01_{-0.04}^{+0.05} \times 10^{36}$ & & - & $1.19_{-0.06}^{+0.07} \times 10^{36}$ & - \\
\hline$\chi^{2} /$ d.o.f & & \multicolumn{2}{|c|}{$382 / 240$} & & $1909 / 671$ & & & $766 / 337$ & \\
\hline
\end{tabular}

As a result, our numbers for $R_{\text {in }}$ should be interpreted with some caution.

In Cluster 2, during intermediate states towards the HIMS/SIMS transition, we find that the jet in MAXI J1659-152 is decreasing both in power and radius of its base, with the disc parameters staying relatively steady. This is roughly consistent with a contracting corona instead of a gradual reduction of the disc truncation radius, which takes place before the state transition (e.g. Fabian et al. 2014; García et al. 2015; Kara et al. 2019). This coronal behaviour is also suggested by recent X-ray variability studies on other similar sources (e.g. Karpouzas et al. 2020, 2021; García et al. 2021). Noticeably, Cluster 2 contains three epochs with order-of-magnitude PC1 differences (Fig. 3d). However, we don't expect our results to be much affected, because it is the hue rather than a single power colour that tracks the outburst evolution (Heil et al. 2015a), and Fig. 4 shows that the hue for all three epochs in Cluster 2 are close to each other, within $2 \sigma$ error range. Therefore, it justifies our choice to jointly fit the epochs that they are at physically similar states. Our joint-fit suggests that the disc is moderately truncated during the transition, implying that the disc inner edge should reduce to the innermost circular orbit (ISCO) (Novikov et al. 1973) only when the source reaches the SIMS/soft state and the jet is quenched.

In Cluster 3, we explore the spectral similarities among multiple BHXB sources sharing nearly identical hues (Fig. 3d). We find a good fit in which the size of the corona $R_{0}$ is tied among epochs, and all three epochs are dominated by the inverse-Comptonization of disc photons. Beyond finding the similarity in the X-ray emitting regions, our fits point to the systems being somewhat different despite sharing similar hue or PSD shapes. In particular, we find that $R_{\text {in }}$ needs to be untied in order to correctly reproduce the data in this cluster. This is not unexpected given how different the SEDs, and particularly the Xray spectra, are from each other. This joint-fit provides evidence that the similarity of the PSD/hue among the BHXB systems is driven by a shared coronal geometry, while the disc truncation radius may not be related to the power colours.

Combining the results from the three joint-fits, we find that the hue and the coronal radius $R_{0}$ seem to follow a clear trend in all of the epoch clusters. Fig. 9 shows a plot $R_{0}$ against hue values from our joint-fits. The figure also includes the results of Lucchini et al. (2021), who modelled multiple HS and HIMS SEDs of MAXI J1836-194 with the same jet model. Both studies show that larger

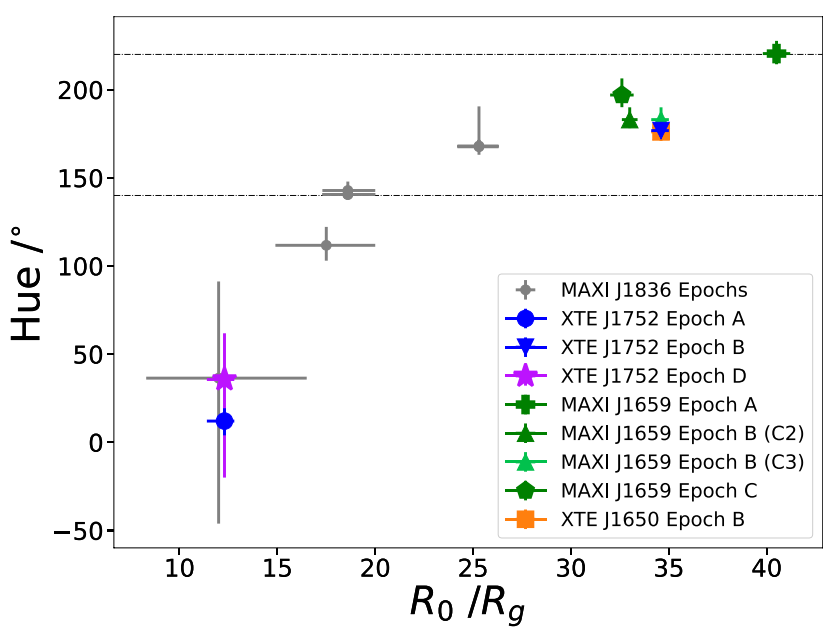

Figure 9. $R_{0}$ versus hue from the joint-fits of this work (coloured) and from Lucchini et al. (2021), who modelled the outburst of MAXI J1836-194 (grey). Dashed lines indicate the hue ranges corresponding to (from bottom to top): HS, HIMS, and SIMS (Heil et al. 2015a). The best-fitting $R_{0}$ of J1659B from both the joint-fits of Cluster 2 (C2, dark green) and Cluster 3 (C3, light green) are plotted.

hues generally correspond to larger $R_{0}$ in both HS and HIMS states. This conclusion is mainly driven by the X-ray data: a natural way of softening the X-ray Comptonization spectrum is to lower the optical depth in the corona by increasing its size; at the same time, in the timing domain softer states usually correspond to a larger powercolour hue. One possible physical interpretation for this trend of increasing $R_{0}$ was proposed by Lucchini et al. (2021): $R_{0}$ can be thought of as a proxy for the radius of the corona-disc boundary at the jet launching region. In GRMHD simulations the size of this region is set by the pressure balance between the gas pressure at the disc/jet interface. Recent simulations show that thinner discs still launch jets, but the jets are less collimated than those launched from thicker discs (Liska et al. 2019). Our findings confirm this $R_{0}$ versus hue trend in multiple BHXB systems and strengthen the suggestion in Lucchini et al. 2021 of a pressure-balanced coronal boundary. Furthermore, our results favour a shared geometrical configuration in the corona among BHXB sources when they evolve to similar 
stages during the outbursts, as also indicated by power spectral studies (e.g. Done \& Kubota 2006; Done et al. 2007; Ingram \& Done 2012). This idea about the BHXB population is demonstrated most strongly in our joint-fit for Cluster 3 , in which all three epochs can be modelled with identical coronal radii, while one epoch (J1650B) is clearly more disc-dominated than the other two epochs. Our work demonstrates the potential of combining the spectral and timing analyses to characterize evolution stages.

Somewhat counter-intuitively, in MAXI J1659-152 the data can be instead fitted by a decreasing $R_{0}$ as the source nears the SIMS and the spectrum softens (Fig. 3 b - although note that in these SEDs, hue and spectral hardness are not well correlated, as shown in Fig. 4). In these SEDs, the joint-fit of Cluster 2 requires both the jet power $N_{j}$ and the electron temperature $T_{e}$ to drop in order to fit the data despite the decrease in $R_{0}$. We can speculate that this drop in $N_{j}$, $R_{0}$, and $T_{e}$ could be due to a gradual weakening of the compact jets, together with an increase in the disc luminosity (leading to an increased cooling of the electrons), before the state transition. This scenario is strengthened by our finding that in this cluster, the jet power $N_{j}$ is dropping as the hue increases. Additionally, as the jet is being quenched, the decrease of its magnetic pressure could help the coronal contraction as well. It is also possible that with the accretion rate increasing towards the Eddington limit, under the increasing radiation pressure, the disc becomes thicker again (e.g. Abramowicz et al. 1988; Abolmasov \& Chashkina 2015; Lančová et al. 2019), resulting again in a strongly collimated jet.

There is a major difference in the X-ray regime between the jointfit of Cluster 1 and the other two joint-fits: in J1752 Epoch A\&D, SSC near the jet base dominates over the scattering of disc photons. This change in the dominant radiative mechanism was also found in MAXI J1836 - 194 (Lucchini et al. 2021), and can be interpreted in terms of the evolution of $R_{0}$, which sets the number density of the radiating electrons: a more compact corona, as is the case for J1752, causes the cyclo-synchrotron radiation energy density $U_{s y n} \propto L_{s y n} / R_{0}^{2}$ to increase, which in turn results in an enhanced SSC emission. Different channels of inverse-Comptonization can potentially cause a difference in the shape of the PSD and thus the hue. This is because the PSD, especially its low-frequency part, is closely related to the coronal response to the fluctuation of the seed spectrum (Uttley et al. 2014, Uttley and Malzac in prep.), and it is unlikely that the accretion rate in the disc and mass loading in the jet base would fluctuate in identical manners. A geometrical change of the accretion disc cannot solely explain the evolution of PSD during BHXB transitions (Ingram \& Done 2011), and our results point to a possible contribution from SSC inside the corona/jet base when the BHXB outburst is in the HS. Together with J1752B and other epochs during the HIMS, our results indicate a shift of the dominant Comptonization channel should take place at some point between the HS and HIMS, due to variation in the coronal and/or truncation radii.

Another main result we find from our joint-fits is that, while large reflection fractions $\operatorname{Refl}_{\text {frac }}$ can be found in epochs regardless of hue, low reflection fractions seem to cluster exclusively in epochs of large hue, near the HIMS/SIMS transition (Fig. 10, although we note that this behaviour is less clear when the spectra are fitted with a phenomenological power-law, rather than our physical model). In the context of a jet model, there are two (not mutually exclusive) mechanisms that could produce this behaviour: an increase in the height of the location of the X-ray emitting region, and an increase in the bulk speed of the jet base, so that jet photons are beamed away from the disc (e.g. Markoff \& Nowak 2004; Dauser et al. 2013). In both of these scenarios, the result would be a lowered fraction of coronal light irradiating the disc and being reprocessed in the reflection spectrum. Recently, X-ray spectral timing-analysis of the BHXB MAXI J1820+ 070 also suggest a similar picture by finding an increasing coronal height towards the HIMS-to-SIMS state transition (Wang et al. 2021; De Marco et al. 2021) (with the caveat that a similar trend is not found in the time-averaged X-ray spectrum). Additionally, based on multi-wavelength variability, Tetarenko et al. (2021) also tentatively propose that the jet bulk Lorenz factor may be an increasing function of the bolometric luminosity, and Wood et al. (2021) find evidence of an increasing ejecta speed of jet blobs as transition nears. Either mechanism is consistent with a model in which the accretion flow gets progressively more magnetically dominated (up to the so-called magnetically arrested disc, or MAD, state) as the accretion rate increases (e.g. Tchekhovskoy, Narayan \& McKinney 2011).

The results of our joint spectral modelling have three main caveats. First, we used the phenomenological convolution model reflect to model the reflected spectrum from the Comptonized continuum in the jet model, rather than a more detailed, stand-alone reflection model (e.g. relxill, Dauser et al. 2014) which accounts for the reflection spectrum more accurately and self-consistently. This choice is because these advanced reflection models cannot yet be easily coupled to our jet model ( $r e l x i l l 1 p$, for example, assumes that the emitting region is a point source, rather than an extended jet nozzle). Nevertheless, we find the trend in the reflection fraction to be in agreement with the picture derived from the detailed X-ray modelling of J1820 (De Marco et al. 2021; Wang et al. 2021), and so we consider our treatment to be a fair approximation of the reflection spectra. Second, we do not include any relativistic ray-tracing effect in our model, meaning that our constraints on the coronal geometry are subject to some systematic uncertainty. In particular, we cannot self-consistently account for variations in the coronal height or its vertical extent easily. These parameters will be explored in a future version of our model.

\section{CONCLUSIONS}

We use the steady-state, multi-zone, semi-analytical jet model bljet to analyse the broadband SEDs of three different BHXB sources: XTE J1752-223, MAXI J1659-152, and XTE J1650-500. We perform joint-fits of epochs with quasi-simultaneous multiwavelength observations at HS and HIMS, clustered according to their timing power spectral colour and the associated hue values. Assuming that all of the non-thermal X-ray emission originates in the corona, associated with the jet base, we find:

(i) Multiple BHXB outbursts in different systems show a common trend in the evolution of the coronal geometry, which can be traced by the power spectral colours. During the transition from the HS to the HIMS, the corona expands due to the decrease in external pressure provided by the disc as it becomes thinner. This expansion results in a lower optical depth in the base of the jets, which in turn causes the Xray spectrum to soften. Our results indicate a change of the dominant $\mathrm{X}$-ray radiative mechanism taken place during this process, from the self-synchrotron Comptonization to the up-scattering of disc thermal photons.

(ii) We also see an indication that the corona then begins to contract close to the HIMS/SIMS transition. We propose that this contraction is related to the jet shutting down. Other possibilities include the disc becomes thicker again under the radiation pressure, re-collimating the jet base. 


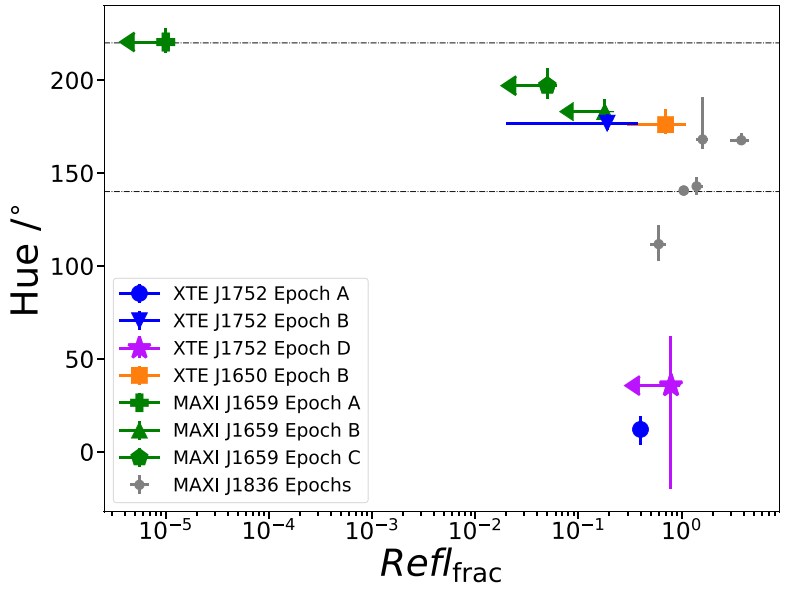

(a)

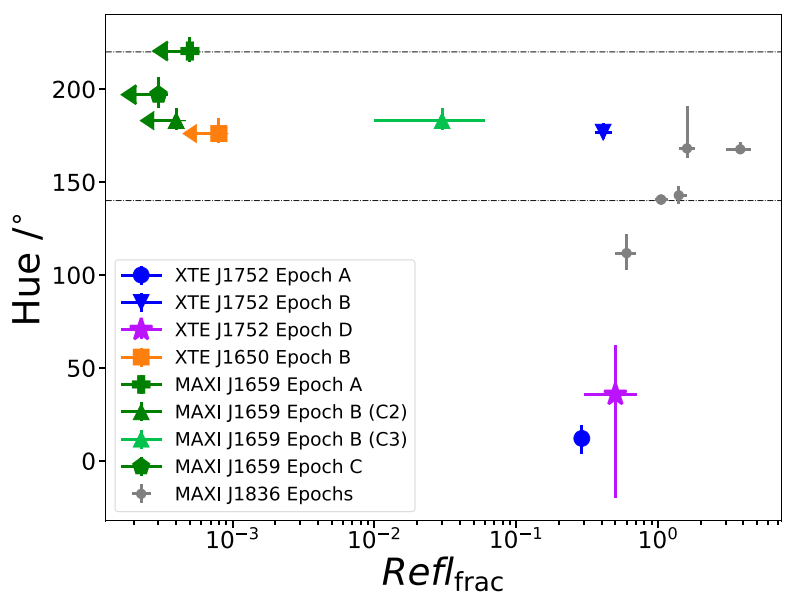

(b)

Figure 10. Refl frac versus hue from the phenomenological X-ray fits (left) and from the broadband SED joint-fits (right). The dashed lines and the colour scheme follows Fig. 9. In both panels we also include the results from the previous study on MAXI J1836-194 (grey, taken from Lucchini et al. 2021). Both phenomenological and physical modelling suggest a decrease in Refl frac as the hue increases.

(iii) While high reflection fractions can be found in all jetted states, from moderately bright hard states up to HIMS/SIMS transition, low reflection fractions seem to be found only near the HIMS/SIMS transition. We propose that this behaviour is caused by an increase in the vertical extent of the corona, and/or if the jet bulk speed increases towards the transition.

\section{ACKNOWLEDGEMENTS}

We thank Dr. Phil Uttley for insightful discussions on X-ray timing analysis. This work has made use of data and software provided by: the High Energy Astrophysics Science Archive Research Center, which is a service of the Astrophysics Science Division at NASA/GSFC; the UK Swift Science Data Centre at the University of Leicester. This work has also made use of the Interactive Spectral Interpretation System maintained by Chandra X-ray Center group at MIT. ML and SM are thankful for support from an NWO (Netherlands Organisation for Scientific Research) VICI award, grant Nr. 639.043.513.

\section{DATA AVAILABILITY}

All data in this paper are publicly available. The radio data were published and tabulated in Corbel et al. (2004), Brocksopp et al. (2013), van der Horst et al. (2013). The infrared, optical, and UV data were published in Curran et al. (2012), van der Horst et al. (2013). The X-ray data are publicly available from HEASARC (ht tps://heasarc.gsfc.nasa.gov/). A reproduction package is available at DOI: 10.5281/zenodo.5002124.

\section{REFERENCES}

Abolmasov P., Chashkina A., 2015, MNRAS, 454, 3432

Abramowicz M., Czerny B., Lasota J., Szuszkiewicz E., 1988, ApJ, 332, 646 Armas Padilla M., Muñoz-Darias T., Sánchez-Sierras J., De Marco B., Jiménez-Ibarra F., Casares J., Corral-Santana J., Torres M., 2019, MNRAS, 485, 5235

Arnaud K., 1996, in Astronomical Data Analysis Software and Systems V. 101, p. 17
Belloni T. M., 2010, in Belloni T., Ed., Lecture Notes in Physics, Vol. 794, States and Transitions in Black Hole Binaries. Springer-Verlag, Berlin, p .53

Beloborodov A. M., 1999, MNRAS, 305, 181

Blandford R., Königl A., 1979, ApJ, 232, 34

Boettcher M., Dermer C. D., 2010, ApJ, 711, 445

Bosch-Ramon V., Romero G. E., Paredes J. M., 2006, A\&A, 447, 263

Brocksopp C., Corbel S., Tzioumis A., Broderick J., Rodriguez J., Yang J., Fender R., Paragi Z., 2013, MNRAS, 432, 931

Brocksopp C., Corbel S., Tzioumis T., Fender R., 2009, Astron. Telegram, 2278, 1

Broderick A. E., Tchekhovskoy A., 2015, ApJ, 809, 97

Cardelli J. A., Clayton G. C., Mathis J. S., 1989, ApJ, 345, 245

Chatterjee K., Liska M., Tchekhovskoy A., Markoff S. B., 2019, MNRAS, 490,2200

Chen W., Shrader C., Livio M., 1997, ApJ, 491, 312

Chun Y. Y. et al., 2013, ApJ, 770, 10

Connors R. et al., 2017, MNRAS, 466, 4121

Connors R. M. et al., 2019, MNRAS, 485, 3696

Connors R. M. et al., 2020, ApJ, 892, 47

Corbel S., Coriat M., Brocksopp C., Tzioumis A., Fender R., Tomsick J., Buxton M., Bailyn C., 2013, MNRAS, 428, 2500

Corbel S., Fender R. P., 2002, ApJ, 573, L35

Corbel S., Fender R. P., Tzioumis A., Nowak M., McIntyre V., Durouchoux P., Sood R., 2000, A\&A, 359, 251

Corbel S., Fender R., Tomsick J., Tzioumis A., Tingay S., 2004, ApJ, 617, 1272

Corbel S., Nowak M., Fender R. P., Tzioumis A., Markoff S., 2003, A\&A, 400, 1007

Corral-Santana J. M., Casares J., Munoz-Darias T., Bauer F. E., Martinez-Pais I. G., Russell D. M., 2016, A\&A, 587, A61

Curran P., Chaty S., Heras J. Z., 2012, A\&A, 547, A41

Dauser T., García J., Parker M., Fabian A., Wilms J., 2014, MNRAS: Letters, 444, L100

Dauser T., Garcia J., Wilms J., Böck M., Brenneman L. W., Falanga M., Fukumura K., Reynolds C. S., 2013, MNRAS, 430, 1694

De Marco B., Zdziarski A., Ponti G., Migliori G., Belloni T., Otero A. S., Dziełak M., Lai E., A\&A, 654, A14

Done C., Gierliński M., Kubota A., 2007, A\&AR, 15, 1

Done C., Kubota A., 2006, MNRAS, 371, 1216

Drappeau S. et al., 2017, MNRAS, 466, 4272

Elvis M., Page C., Pounds K., Ricketts M., Turner M., 1975, Nature, 257, 656 
Esin A. A., McClintock J. E., Narayan R., 1997, ApJ, 489, 865

Evans P. et al., 2009, MNRAS, 397, 1177

Fabian A., Parker M., Wilkins D., Miller J., Kara E., Reynolds C., Dauser T., 2014, MNRAS, 439, 2307

Fabian A., Ross R., 2010, Space Sci. Rev., 157, 167

Falcke H., Körding E., Markoff S., 2004, A\&A, 414, 895

Fender R. et al., 1999, ApJ, 519, L165

Fender R. P., Belloni T. M., Gallo E., 2004, MNRAS, 355, 1105

Fender R., Maccarone T., Van Kesteren Z., 2005, MNRAS, 360, 1085

Fender R., Pooley G., Durouchoux P., Tilanus R., Brocksopp C., 2000, MNRAS, 312, 853

Foight D. R., Güver T., Özel F., Slane P. O., 2016, ApJ, 826, 66

Foreman-Mackey D., Hogg D. W., Lang D., Goodman J., 2013, PASP, 125 , 306

Fürst F. et al., 2015, ApJ, 808, 122

Gallo E. et al., 2014, MNRAS, 445, 290

Gallo E., Fender R. P., Pooley G., 2003, MNRAS, 344, 60

Gallo E., Migliari S., Markoff S., Tomsick J. A., Bailyn C. D., Berta S., Fender R., Miller-Jones J. C., 2007, ApJ, 670, 600

Gandhi P. et al., 2008, MNRAS: Letters, 390, L29

García F., Méndez M., Karpouzas K., Belloni T., Zhang L., Altamirano D., 2021, MNRAS, 501, 3173

García J. A. et al., 2018, ApJ, 864, 25

García J. A., Steiner J. F., McClintock J. E., Remillard R. A., Grinberg V., Dauser T., 2015, ApJ, 813, 84

Gierliński M., Done C., Page K., 2009, MNRAS, 392, 1106

Haardt F., Maraschi L., 1993, ApJ, 413, 507

Haardt F., Maraschi L., Ghisellini G., 1994, ApJ, 432, L95

Hannikainen D., Hunstead R., Campbell-Wilson D., Sood R., 1998, A\&A, 337,460

Heil L., Uttley P., Klein-Wolt M., 2015a, MNRAS, 448, 3339

Heil L., Uttley P., Klein-Wolt M., 2015b, MNRAS, 448, 3348

Hjellming R., Johnston K., 1988, ApJ, 328, 600

Homan J., Belloni T., 2005, in Astrophysics and Space Science. SpringerVerlag, Berlin, 300, p. 107

Homan J., Wijnands R., Kong A., Miller J. M., Rossi S., Belloni T., Lewin W. H., 2006, MNRAS, 366, 235

Houck J., Denicola L., 2000, in Nadine M., Christian V., Dennis C., eds ASP Conf. Ser. Vol. 216, Astronomical Data Analysis Software and Systems IX. Astron. Soc. Pac., San Francisco, p. 591

Ingram A. R., Motta S. E., 2019, New Astron. Rev., 85, 101524

Ingram A., Done C., 2011, MNRAS, 415, 2323

Ingram A., Done C., 2012, MNRAS, 419, 2369

Jahoda K., Swank J. H., Giles A. B., Stark M. J., Strohmayer T., Zhang W. W., Morgan E. H., 1996, in Oswald H.S., Mark A.G., eds, Proc. SPIE Conf. Ser. Vol. 2808, EUV, X-Ray, and Gamma-Ray Instrumentation for Astronomy VII. SPIE, Bellingham, 59

Jonker P. G., Miller-Jones J. C., Homan J., Tomsick J., Fender R., Kaaret P., Markoff S., Gallo E., 2012, MNRAS, 423, 3308

Kalamkar M., Homan J., Altamirano D., Van Der Klis M., Casella P., Linares M., 2011, ApJ, 731, L2

Kantzas D. et al., 2021, MNRAS, 500, 2112

Kara E. et al., 2019, Nature, 565, 198

Karpouzas K., Méndez M., García F., Zhang L., Altamirano D., Belloni T., Zhang Y., 2021, MNRAS, 503, 5522

Karpouzas K., Méndez M., Ribeiro E. M., Altamirano D., Blaes O., García F., 2020, MNRAS, 492, 1399

Kennea J., Krimm H., Mangano V., Curran P., Romano P., Evans P., Burrows D., 2010, Astron. Telegram, 2877,

Klein-Wolt M., Van Der Klis M., 2008, ApJ, 675, 1407

Kong A. K., 2012, ApJ, 760, L27

Kuulkers E. et al., 2013, A\&A, 552, A32

Kylafis N., Contopoulos I., Kazanas D., Christodoulou D., 2012, A\&A, 538, A5

Lančová D. et al., 2019, ApJ, 884, L37

Liska M., Tchekhovskoy A., Ingram A., Van Der Klis M., 2019, MNRAS, 487,550
Lucchini M., Markoff S., Crumley P., Krauß F., Connors R., 2019, MNRAS, 482, 4798

Lucchini M., Russell T. D., Markoff S. B., Vincentelli F., Gardenier D., Ceccobello C., Uttley P., 2021, MNRAS, 501, 5910

Magdziarz P., Zdziarski A. A., 1995, MNRAS, 273, 837

Maitra D., Markoff S., Brocksopp C., Noble M., Nowak M., Wilms J., 2009, MNRAS, 398, 1638

Maitra D., Miller J. M., Markoff S., King A., 2011, ApJ, 735, 107

Malzac J., 2013, MNRAS: Letters, 429, L20

Malzac J., 2014, MNRAS, 443, 299

Mangano V., Hoversten E., Markwardt C., Sbarufatti B., Starling R., Ukwatta T., 2010, GCN, 11296,

Markoff S. et al., 2008, ApJ, 681, 905

Markoff S. et al., 2015, ApJ, 812, L25

Markoff S., Falcke H., Fender R., 2001a, A\&A, 372, L25

Markoff S., Falcke H., Yuan F., Biermann P. L., 2001b, A\&A, 379, L13

Markoff S., Nowak M. A., 2004, ApJ, 609, 972

Markoff S., Nowak M. A., Wilms J., 2005, ApJ, 635, 1203

Markwardt C. et al., 2009, Astron. Telegram, 2258, 1

Martocchia A., Matt G., 1996, MNRAS, 282, L53

Matt G., Perola G., Piro L., Stella L., 1992, A\&A, 257, 63

McHardy I., Koerding E., Knigge C., Uttley P., Fender R., 2006, Nature, 444, 730

Méndez M., Belloni T., van der Klis M., 1998, ApJ, 499, L187

Merloni A., Fabian A., 2002, MNRAS, 332, 165

Merloni A., Heinz S., Di Matteo T., 2003, MNRAS, 345, 1057

Miller J. et al., 2002, ApJ, 570, L69

Miller J. et al., 2015, ApJ, 799, L6

Miller-Jones J., Madej O., Jonker P., Homan J., Ratti E., Torres M., 2011, Astron. Telegram, 3358, 1

Miniutti G., Fabian A., Miller J., 2004, MNRAS, 351, 466

Molla A. A., Debnath D., Chakrabarti S. K., Mondal S., Jana A., 2016, MNRAS, 460, 3163

Montanari E., Titarchuk L., Frontera F., 2009, ApJ, 692, 1597

Mościbrodzka M., Gammie C. F., Dolence J. C., Shiokawa H., 2011, ApJ, 735,9

Narayan R., Yi I., 1994, ApJ, 428, L13

Negoro H. et al., 2010, Astron. Telegram, 2873, 1

Neronov A., Aharonian F. A., 2007, ApJ, 671, 85

Novikov I., Thorne K., Dewitt C., Dewitt B., 1973, Gordon Breach Sci. Publ., New York, p. 343

Nowak M. A., 1995, PASP, 107, 1207

Orosz J. A., McClintock J. E., Remillard R. A., Corbel S., 2004, ApJ, 616, 376

Plotkin R. M., Markoff S., Kelly B. C., Körding E., Anderson S. F., 2012, MNRAS, 419, 267

Rapisarda S., Ingram A., van der Klis M., 2017, MNRAS, 472, 3821

Ratti E. et al., 2012, MNRAS, 423, 2656

Remillard R. A., McClintock J. E., 2006, Annu. Rev. Astron. Astrophys., 44, 49

Remillard R., 2001, IAUC, 7707, 1

Romero G. E., Orellana M., 2005, A\&A, 439, 237

Russell D. et al., 2013, MNRAS, 429, 815

Shakura N. I., Sunyaev R. A., 1973, A\&A, 24, 337

Shakura N., Sunyaev R., 1976, MNRAS, 175, 613

Shang J.-R., Debnath D., Chatterjee D., Jana A., Chakrabarti S., Chang H.-K., Yap Y.-X., Chiu C.-L., 2019, ApJ, 875, 4

Shaposhnikov N., Markwardt C., Swank J., Krimm H., 2010, ApJ, 723, 1817

Slanỳ P., Stuchlik Z., 2008, A\&A, 492, 319

Tanaka Y., Shibazaki N., 1996, Annu. Rev. Astron. Astrophys., 34, 607

Tananbaum H., Gursky H., Kellogg E., Giacconi R., Jones C., 1972, ApJ, 177, L5

Tchekhovskoy A., Narayan R., McKinney J. C., 2011, MNRAS: Letters, 418, L79

Tetarenko A. et al., 2021, MNRAS, 504, 3862

Tetarenko B., Sivakoff G., Heinke C., Gladstone J., 2016, ApJS, 222, 15

Uttley P. et al., 2018, Astron. Telegram, 11423, 1

Uttley P., Cackett E., Fabian A., Kara E., Wilkins D., 2014, A\&AR, 22, 1 
van der Horst A. et al., 2013, MNRAS, 436, 2625

Van der Klis M., 1989, in Ögelman H., van den Heuvel E. P. J., eds, Timing neutron stars. Kluwer Academic, New York, p. 27

Veledina A., Poutanen J., Vurm I., 2013, MNRAS, 430, 3196

Wang J. et al., 2021, ApJ, 910, L3

Wenger M. et al., 2000, Astronomy and Astrophysics Supplement Series, 143,9

Wijnands R., van der Klis M., 1999, ApJ, 514, 939

Wilms J., Allen A., McCray R., 2000, ApJ, 542, 914

Wood C. et al., 2021, MNRAS, 505, 3393

Xu Y., Harrison F. A., Tomsick J. A., Walton D. J., Barret D., García J. A., Hare J., Parker M. L., 2020, ApJ, 893, 30

Yuan F., Zdziarski A. A., Xue Y., Wu X.-B., 2007, ApJ, 659, 541

Zdziarski A. A., De Marco B., Szanecki M., Niedźwiecki A., Markowitz A., 2021, ApJ, 906, 69

\section{APPENDIX A: APPENDIX}

\section{XTE J1752-223}

The X-ray transient XTE J1752-223 was discovered by RXTE in 2009 October (Markwardt et al. 2009) when it went into an outburst, lasting for almost 8 months; the evolution of the outburst was typical of that of a black hole system, as shown in Fig. 3a. The radio emission is consistent with a typical compact jet in the hard state and shows optically thin flares in the soft states (Brocksopp et al. 2013). The mass of the black hole is estimated to be $9.6 \pm 0.8 M_{\odot}$, using correlations between spectral and variability properties with GRO J1655-40 and XTE J1550-564 (Shaposhnikov et al. 2010), while there is a lack of dynamical mass constraint. From detailed modelling of the reflection signatures in the X-ray spectra for the monthlong high-hard state with stable X-ray luminosity and hardness, the inclination angle of XTE J1752-223 is estimated to be $35^{\circ} \pm 4^{\circ}$, and the Galactic extinction $N_{H}$ to be $1.0 \times 10^{22} \mathrm{~cm}^{-2}$ (García et al. 2018).

The distance $d$ to XTE J1752-223 is not well constrained. A distance of $3.5 \pm 0.4 \mathrm{kpcs}$ is determined by Shaposhnikov et al. (2010) using the same technique for mass estimation. However, modelling of X-ray photoelectric absorption edges in the source (Chun et al. 2013) suggests a larger distance (>5 kpcs), and the optical detection on the companion star during the quiescence of XTE J1752-223 (Ratti et al. 2012) also favours a larger distance up to $8 \mathrm{kpcs}$. A distance larger than $3.5 \mathrm{kpcs}$ also agrees with the high column density $N_{H}$ from X-ray spectroscopy; at a distance of 3.5 kpcs on the same line of sight towards XTE J1752-223, the Galactic extinction is about 60 per cent of what is suggested by X-ray studies (Chun et al. 2013). Therefore, in this work we take $6 \mathrm{kpcs}$ as the distance of XTE J1752-223 (Ratti et al. 2012).

RXTE monitored XTE J1752-223 throughout the entire 20092010 outburst. In this work, we only analyse $R X T E$ data taken on four dates (year-month-day: 2009-11-05, 2010-01-21, 2010-04-14, 201006-03), highlighted by red circles in the HID in Fig. 3a. These four dates correspond to epochs in which the compact jet is detected by Australia Telescope Compact Array (ATCA) (Brocksopp et al. 2009). All the radio and X-ray observations of XTE J1752-223 used in this work are listed in Table A1 and Table A2, and the quasi-simultaneous
UV data for XTE J1752-223 is provided by Swift/UVOT, while $\mathrm{Swift} / X R T$ provides the soft $\mathrm{X}$-ray spectra down to $1 \mathrm{keV}$.

\section{MAXI J1659-152}

MAXI J1659-152 was detected by Swift and MAXI during its 2010 outburst (Mangano et al. 2010; Negoro et al. 2010). Its orbital period of $\sim 2.4$ h (Kuulkers et al. 2013) makes it the shortest period BHXB source known (Kennea et al. 2010). In this work, we take $6_{-1.3}^{+1.8} M_{\odot}$ as the black hole mass, estimated by Molla et al. (2016). The inclination angle of the system is constrained to be $65^{\circ}<i<80^{\circ}$ by using the cyclical absorption dips in X-ray lightcurves (Kuulkers et al. 2013). In this work, we take $i=75^{\circ}$.

Like XTE J1752-223, the distance to MAXI J1659-152 is poorly constrained, ranging from 1.6 to $8.6 \mathrm{kpcs}$ (Kennea et al. 2010; MillerJones et al. 2011; Jonker et al. 2012; Kong 2012; Kuulkers et al. 2013). Here we take it to be $6 \mathrm{kpcs}$. This value reconciles different estimations and agrees with Kong 2012 who suggest the companion star is an M2 dwarf.

In van der Horst et al. (2013) they analyse multi-wavelength data of the entire outburst, lasting about $40 \mathrm{~d}$. These authors estimates a line-of-sight column density $N_{H}=(0.319 \pm 0.009) \times 10^{22} \mathrm{~cm}^{-2}$. The multi-wavelength data are presented in Table A3 and Table A4. The three epochs in which radio, IR/optical/UV and X-ray data are available and our jet model is applicable, are selected and highlighted by red circles in Fig. 3b. The X-ray observations used in the spectral analysis by this work are listed in Table A5). Within the selected time interval, the jet break frequency moved from IR to radio band (van der Horst et al. 2013), which can be an indication of the jet particle acceleration region extending further out from the black hole (Lucchini et al. 2021, also see the model section).

\section{XTE J1650-500}

XTE J1650-500 was first discovered by RXTE during its 2001 outburst (Remillard 2001). We adopt 5.1 $M_{\odot}$ for the mass of the black hole, as estimated by Slany \& Stuchlik (2008) and within the mass limit estimated by Orosz et al. (2004) $\left(2.7<M_{B H}<7.3 M_{\odot}\right)$. We take an intermediate inclination $i=45^{\circ}$, following estimates from modelling its X-ray reflection spectra (Miller et al. 2002; Miniutti et al. 2004). The distance of XTE J1650-500 is estimated to be $2.6 \pm 0.7 \mathrm{kpcs}$ by empirically studying the X-ray luminosity of BHXBs during the state transitions (Homan et al. 2006). $N_{H}$ estimations vary among studies but all favour a moderate absorption (e.g. Miller et al. 2002; Montanari, Titarchuk \& Frontera 2009); here we take $N_{H}=(0.5 \pm 0.1) \times 10^{22} \mathrm{~cm}^{-2}$ from (Miniutti et al. 2004).

Fig. $3 \mathrm{c}$ shows the path of the source on the HID during its outburst, using RXTE data. In total there are eight radio observations simultaneous with X-ray observations, but only two epochs have simultaneous IR data at the HS or HIMS, indicated by red circles in Fig. 3c. Note that we only analyse RXTE observations of these two epochs mentioned above. Multi-wavelength spectral analysis (radio/O/IR/X-ray) is only performed on the two highlighted epochs. See Table A6, Table A7, and Table A8 for the lists of radio, IR, and $\mathrm{X}$-ray observations used in this work respectively. 
Table A1. List of radio observations of XTE J1752-223 analysed in this work, detected by ATCA. Flux data in units of mJy in two frequency bands are adopted from Brocksopp et al. (2013) and labelled according to their corresponding X-ray epochs.

\begin{tabular}{lcccc}
\hline Date & MJD & $5.5 \mathrm{GHz}(\mathrm{mJy})$ & $9 \mathrm{GHz}(\mathrm{mJy})$ & Epoch \\
\hline $2009-11-05$ & 55140.4 & $1.87 \pm 0.07$ & $2.05 \pm 0.07$ & $\mathrm{~A}$ \\
$2010-01-21$ & 55217.9 & $20.00 \pm 0.06$ & $21.71 \pm 0.04$ & $\mathrm{~B}$ \\
$2010-04-14$ & 55300.9 & $1.07 \pm 0.09$ & $0.09 \pm 0.05$ & $\mathrm{C}$ \\
$2010-06-03$ & 55350.5 & $0.20 \pm 0.03$ & $0.18 \pm 0.03$ & $\mathrm{D}$ \\
\hline
\end{tabular}

Table A2. List of X-ray observations of XTE J1752-223 analysed in this work, with associated epochs labelled. The UV flux of each epoch is derived from Swift $U V O T$ images taken during the same observations listed. On 2009-11-05, RXTE took three successive observations and in this work, we only study the first one for spectral analysis, which is the closest to the radio observation in time on the same day, but include the rest two in power-colour timing analysis. Swift has no observations coinciding Epoch B, due to the orbital restraints of the satellite.

\begin{tabular}{lccc}
\hline Satellite & Date & ObsID & Epoch \\
\hline RXTE & $2009-11-05$ & $94331-01-02-11$ & A \\
& $2010-01-21$ & $94331-01-06-02$ & B \\
& $2010-04-13$ & $95360-01-12-03$ & C \\
Swift & $2010-06-03$ & $95702-01-07-03$ & D \\
& $2009-11-03$ & 00031532009 & A \\
& $2010-04-14$ & 00031688002 & C \\
\hline
\end{tabular}

Table A3. List of radio observations of MAXI J1659-152 analysed in this work, detected by Very Large Array (VLA). Flux data adopted from van der Horst et al. (2013), labelled with Epoch A/B/C.

\begin{tabular}{lcccccc}
\hline Date & MJD & $4.9 \mathrm{GHz}(\mathrm{mJy})$ & $8.5 \mathrm{GHz}(\mathrm{mJy})$ & $22 \mathrm{GHz}(\mathrm{mJy})$ & $43 \mathrm{GHz}(\mathrm{mJy})$ & Epoch \\
\hline $2010-09-29$ & 55468.05 & $9.88 \pm 0.30$ & $10.03 \pm 0.31$ & $11.81 \pm 0.71$ & $11.19 \pm 0.59$ & A \\
$2010-10-01$ & 55470.06 & $10.29 \pm 0.32$ & $9.74 \pm 0.30$ & $8.84 \pm 0.49$ & $4.84 \pm 0.35$ & $\mathrm{~B}$ \\
$2010-10-03$ & 55461.98 & $9.23 \pm 0.28$ & $7.55 \pm 0.42$ & $7.88 \pm 0.42$ & $3.74 \pm 0.40$ \\
\hline
\end{tabular}

Table A4. List of IR/UV observations of MAXI J1659-152 used in this work. The magnitude of the data in each filter without de-reddening is adopted from van der Horst et al. (2013). Data are de-reddened (See text above) before the spectral analysis.

\begin{tabular}{|c|c|c|c|c|c|c|}
\hline Date & MJD & Instrument & Filter & Magnitude & Error & Epoch \\
\hline 2010-09-29 & 55468.05 & SMARTS & $\mathrm{H}$ & 14.7 & 0.13 & A \\
\hline \multirow[t]{3}{*}{ 2010-10-01 } & 55469.79 & BOOTES-2 & $\mathrm{R}$ & 16.59 & 0.06 & $\mathrm{~B}$ \\
\hline & 55469.993 & SMARTS & $\mathrm{J}$ & 15.26 & 0.1 & B \\
\hline & 55469.999 & SMARTS & $\mathrm{H}$ & 14.88 & 0.21 & B \\
\hline \multirow{2}{*}{ 2010-10-03 } & 55472.002 & SMARTS & $\mathrm{H}$ & 15.02 & 0.08 & $\mathrm{C}$ \\
\hline & 55472.12 & UVOT & UVW1 & 16.317 & 0.027 & $\mathrm{C}$ \\
\hline
\end{tabular}

Table A5. List of X-ray observations of MAXI J1659-152 downloaded, reduced, and analysed in this work. From 2010-09-29 to 2010-10-15 only a fraction of the RXTE observations has science event observation modes available for power colour analysis (see text), which is all included in this work. Timing analysis has been performed on all the listed observations in order to track the power colour evolution, while spectral analysis only considers the observations that coincide with the radio/IR/UV observations (the closest if there are multiple observations on the same day), labelled by Epoch A/B/C.

\begin{tabular}{lccc}
\hline Satellite & Date & ObsID & Epoch \\
\hline RXTE & $2010-09-29$ & $95358-01-02-01$ & A \\
& $2010-10-01$ & $95108-01-02-00$ & B \\
Swift & $2010-10-03$ & $95108-01-05-00$ & C \\
& $2010-09-29$ & 00434928007 & A \\
& $2010-10-01$ & 00434928009 & B \\
\hline
\end{tabular}


Table A6. List of radio observations of XTE J1650-500 used in this work. All flux density data is in units of mJy, adopted from Corbel et al. (2004) and labelled by Epoch A/B.

\begin{tabular}{lcccccc}
\hline Date & MJD & $1384 \mathrm{MHz}$ & $2496 \mathrm{MHz}$ & $4800 \mathrm{MHz}$ & $8640 \mathrm{MHz}$ & Epoch \\
\hline $2001-09-08$ & 52160.81 & $4.08 \pm 0.20$ & $5.30 \pm 0.15$ & $5.28 \pm 0.10$ & $4.48 \pm 0.10$ \\
$2001-09-24$ & 52177.01 & - & - & $0.83 \pm 0.10$ & $0.77 \pm 0.10$ & A \\
\hline
\end{tabular}

Table A7. List of IR observations of XTE J1650-500 used in this work. The data magnitudes before de-reddening are adopted from Curran et al. (2012). The data are de-reddened before the spectral analysis.

\begin{tabular}{|c|c|c|c|c|c|}
\hline Date & MJD & Filter & Magnitude & Error & Epoch \\
\hline 2001-09-08 & 52161.03 & $\mathrm{H}$ & 13.79 & 0.11 & A \\
\hline \multirow[t]{3}{*}{ 2001-09-25 } & 52176.99 & $\mathrm{~J}$ & 14.65 & 0.09 & B \\
\hline & 52176.99 & $\mathrm{H}$ & 14.16 & 0.12 & B \\
\hline & 52177.00 & $K_{S}$ & 13.82 & 0.12 & B \\
\hline
\end{tabular}

Table A8. List of X-ray observations of XTE J1650-500 downloaded, reduced, and analysed in this work. For this source in X-ray, only RXTE data were analysed. Timing analysis has been performed on all the listed observations in order to track the power colour evolution, while spectral analysis only considers the observations that coincide with the radio/IR/UV observations, labelled by Epoch A/B.

\begin{tabular}{lcc}
\hline Date & ObsID & Epoch \\
\hline $2001-09-08$ & $60113-01-03-00$ & A \\
$2001-09-25$ & $60113-01-19-00$ & B \\
\hline
\end{tabular}

Table A9. Best-fitting parameters of clusters considered in this work. Similar settings as Table 4, but with $R_{0}$ and $R_{\text {in }}$ untied among all the epochs.

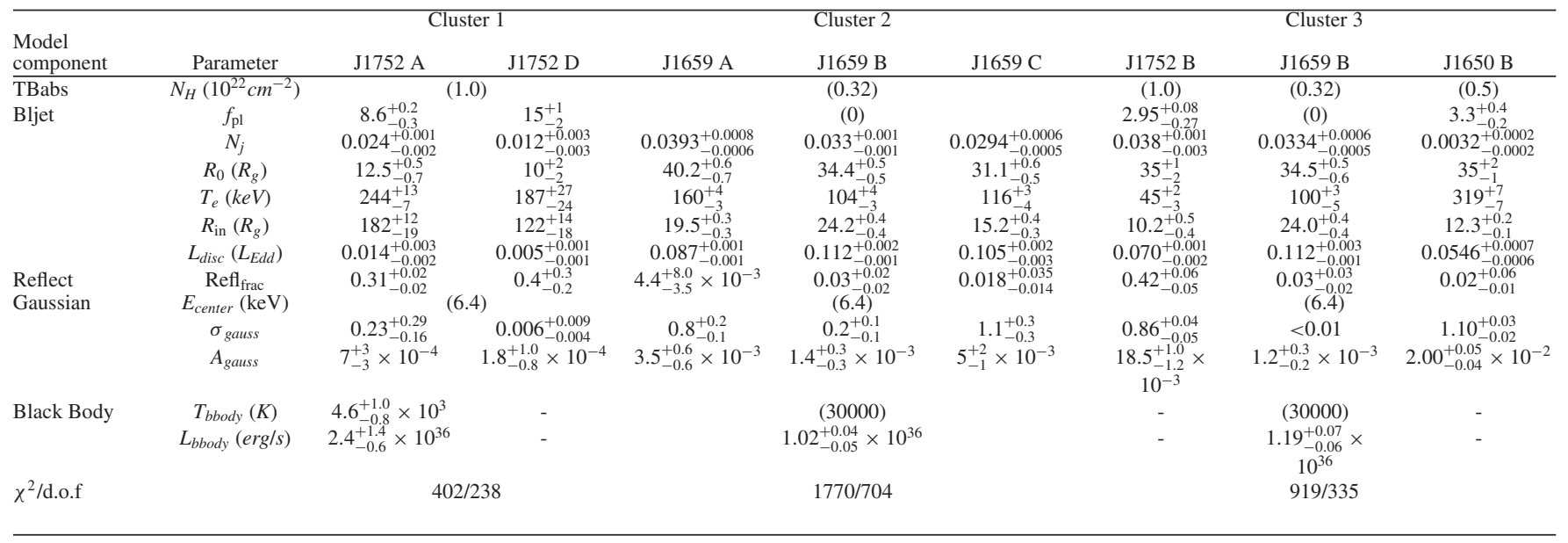

This paper has been typeset from a TEX/LTEX file prepared by the author. 